\title{
LIMIT LINEAR SERIES FOR CURVES NOT OF COMPACT TYPE
}

\author{
BRIAN OSSERMAN
}

\begin{abstract}
We introduce a notion of limit linear series for nodal curves which are not of compact type. We give a construction of a moduli space of limit linear series, which works also in smoothing families, and we prove a corresponding specialization result. For a more restricted class of curves which simultaneously generalizes two-component curves and curves of compact type, we give an equivalent definition of limit linear series, which is visibly a generalization of the Eisenbud-Harris definition. Finally, for the same class of curves, we prove a smoothing theorem which constitutes an improvement over known results even in the compact-type case.
\end{abstract}

\section{INTRODUCTION}

The 1980's saw spectacular progress in the theory of linear series on curves and their applications, including the proofs of the Brill-Noether (Griffiths-Harris [GH80]) and Gieseker-Petri (Gieseker [Gie82]) theorems, new results on the geometry of general linear series (Eisenbud-Harris [EH83]), and the proof that moduli spaces of curves of sufficiently high genus are of general type (Harris-Mumford [HM82] and Eisenbud-Harris [EH87]). What these results all had in common was that they made central use of degeneration techniques, studying what happens to linear series as smooth curves degenerate to singular ones. Ultimately, Eisenbud and Harris developed a general theory of "limit linear series" for curves of compact type, meaning those curves whose dual graphs are trees, or equivalently, whose Jacobians are compact.

For more than 25 years, the question of how to extend the Eisenbud-Harris theory to curves not of compact type has remained open. Aside from the intrinsic appeal of the question, there are various reasons one would like to have such a theory:

- it would offer the most systematic approach to computing the cohomology classes of higher-codimension Brill-Noether classes on moduli spaces of curves;

- it would allow greater flexibility in choosing a degeneration to approach open questions such as the maximal rank conjecture;

- and it likewise offers a more general setting for analyzing generic fibers of specific families of curves. For instance, degenerations arising from considering modular curves in positive characteristic are often two-component nodal curves.

The question of limit linear series for curves not of compact type has been explored by Esteves in various papers, most notably with Medeiros in [EM02], but to date,

The author was partially supported by NSA grant H98230-11-1-0159 and Simons Foundation grant \#279151 during the preparation of this work. 
no one has been able to develop a complete theory generalizing that of Eisenbud and Harris. Recently, Amini and Baker [AB] have proposed a notion of limit linear series based on Brill-Noether theory for graphs, which they show generalizes the definition of Eisenbud and Harris. However, while they prove a specialization theorem, it is not clear how to prove a smoothing theorem for Amini-Baker limit linear series, or how to construct moduli spaces.

In the present paper, we propose a different notion of limit linear series for curves not of compact type, developed independently and motivated in part by work of the author in higher rank [Oss14c]. After giving the definition, we construct moduli spaces both over individual curves and in smoothing families, and use them to prove a specialization result. We then show that our definition is a generalization of the Eisenbud-Harris definition. In fact, we do considerably more: for the class of curves of "pseudocompact type," which is a simultaneous generalization of curves of compact type and curves with two components (see Figure 1 below), we give an equivalent formulation which visibly generalizes the Eisenbud-Harris definition. In essence, our more general definition is well-suited for abstract theory and constructions, while the second definition is more tractable for computations. Finally, for curves of pseudocompact type we prove a smoothing theorem, which is an improvement even for the compact-type case because it does not only apply to refined limit linear series.

To apply our smoothing theorem, it is necessary to produce families of limit linear series having the expected dimension, and accordingly in [Oss14a] we carry out dimension counts. Using our generalized Eisenbud-Harris definition, we show that for curves of pseudocompact type the expected dimension of spaces of limit linear series is always correct, in the sense that if certain gluing conditions impose the maximal codimension, then the dimension agrees with the Brill-Noether number $\rho$. We also investigate several families of curves for which we can show the gluing conditions do indeed impose the maximal codimension, giving in particular new criteria for the generic fiber of a one-parameter family of curves to be Brill-Noether general. One of the families we consider in [Oss14a] is a broad generalization of the curves considered by Cools, Draisma, Payne and Robeva in the graph-theoretic context in [CDPR12], and we are able to use our theory to shed new light on their results, and to suggest further directions of investigation for the Brill-Noether theory of graphs. The relationship to the Amini-Baker theory will be investigated more thoroughly in [Oss14b], but in essence our approach keeps track of more gluing data, while minimizing the role of graph theory. Although this may in principle make computations more difficult, in practice this may not be the case, and we have found that our approach has the desired dimension behavior in some cases (such as binary curves) for which the Amini-Baker theory does not.

We now explain the basic ideas that go into our definition of limit linear series. Suppose that $B=\operatorname{Spec} R$ with $R$ a discrete valuation $\operatorname{ring}$, and $X \rightarrow B$ is a family of curves over $B$ with smooth generic fiber and reducible nodal special fiber $X_{0}$. Further suppose that the total space $X$ is regular. Then each component $Z_{v}$ of $X_{0}$ is a (Cartier) divisor on $X$, so any given extension of a line bundle $\mathscr{L}_{\eta}$ on the generic fiber can be twisted by $\mathscr{O}_{X}\left(Z_{v}\right)$ to obtain an infinite family of extensions. Given $V_{\eta}$ an $(r+1)$-dimensional space of global sections of $\mathscr{L}_{\eta}$, for any extension of $\mathscr{L}_{\eta}$ there is a unique extension of $V_{\eta}$. The idea introduced by Eisenbud and Harris was to use these twists to concentrate multidegree on each component $Z_{v}$ of $X_{0}$, 
and then to restrict the resulting extension of $\left(\mathscr{L}_{\eta}, V_{\eta}\right)$ to $Z_{v}$, thereby obtaining a collection $\left(\mathscr{L}^{v}, V^{v}\right)_{v}$ of $\mathfrak{g}_{d}^{r}$ s on the components of $X_{0}$. The question then becomes to understand which such tuples of $\mathfrak{g}_{d}^{r} \mathrm{~s}$ can arise as a limit in this way. Eisenbud and Harris found a compatibility condition in terms of vanishing sequences at the nodes, and used this to define their notion of limit linear series. The power of their definition was that it was fundamentally inductive, describing limit linear series almost independently on each component, and thereby making computations very tractable. However, the drawback of their definition was that it was difficult to generalize, and also to use for more theoretical purposes, such as moduli space constructions.

In [Oss14c], it was shown that one can state an equivalent formulation of the Eisenbud-Harris definition as follows: if $w$ is a multidegree of total degree $d$ on $X_{0}$, and $\mathscr{L}_{w}$ denotes the extension of $\mathscr{L}_{\eta}$ having multidegree $w$, then $\Gamma\left(X_{0},\left.\mathscr{L}_{w}\right|_{X_{0}}\right)$ contains the extension of $V_{\eta}$, and must therefore have dimension at least $r+1$. Moreover, it is straightforward to see that this extension of $V_{\eta}$ may be obtained by gluing together sections from the various $V^{v}$. This leads to a definition of limit linear series as a generalized determinantal locus (Definition 2.21 below), which yields new moduli space constructions, and which also lends itself to generalization to curves not of compact type. The other basic ingredient of our definition is that we allow for insertion of chain of rational curves at nodes, and keep track of information on these curves as combinatorially as possible, only considering spaces of global sections on the original components.

We next discuss our equivalent definition, generalizing the Eisenbud-Harris definition to a broader class of curves. We begin by recalling their definition. Given a tuple $\left(\mathscr{L}^{v}, V^{v}\right)$ of $\mathfrak{g}_{d}^{r} \mathrm{~s}$ on the components $Z_{v}$ of $X_{0}$, Eisenbud and Harris define the tuple to be a limit linear series if the following condition is satisfied: for every node of $X_{0}$, given as $Z_{v} \cap Z_{v^{\prime}}$, write $a_{0}^{v}, \ldots, a_{r}^{v}$ and $a_{0}^{v^{\prime}}, \ldots, a_{r}^{v^{\prime}}$ for the vanishing sequences of $\left(\mathscr{L}^{v}, V^{v}\right)$ (respectively, $\left(\mathscr{L}^{v^{\prime}}, V^{v^{\prime}}\right)$ ) at the node in question; then we require

$$
a_{j}^{v}+a_{r-j}^{v^{\prime}} \geqslant d
$$

for $j=0, \ldots, r$.

Our generalized definition builds on this by replacing the vanishing sequence with a "multivanishing sequence" which keeps track of vanishing at several points at a time, and by adding a gluing condition on the spaces $V^{v}$, which is vacuously satisfied in the compact type case. There are additional complications arising from keeping track of potential chains of rational curves inserted at each node, but we illustrate the main ideas in the simplest case, where we have two components, and do not insert any additional rational curves.

Some preliminary definitions are as follows.

Notation 1.1. Let $X$ be a smooth projective curve, $D$ an effective divisor on $X$, and $(\mathscr{L}, V)$ a $\mathfrak{g}_{d}^{r}$ on $X$. Then we denote by $V(-D)$ the space $V \cap H^{0}(X, \mathscr{L}(-D))$.

Definition 1.2. Let $X$ be a smooth projective curve, $r, d \geqslant 0$, and $D_{0} \leqslant D_{1} \leqslant$ $\cdots \leqslant D_{b+1}$ a sequence of effective divisors on $X$, with $D_{0}=0$ and $\operatorname{deg} D_{b+1}>d$. Given $(\mathscr{L}, V)$ a $\mathfrak{g}_{d}^{r}$ on $X$, define the multivanishing sequence of $(\mathscr{L}, V)$ along $D$. to be the sequence

$$
a_{0} \leqslant \cdots \leqslant a_{r}
$$


where a value $a$ appears in the sequence $m$ times if for some $i$ we have $\operatorname{deg} D_{i}=a$, $\operatorname{deg} D_{i+1}>a$, and $\operatorname{dim}\left(V\left(-D_{i}\right) / V\left(-D_{i+1}\right)\right)=m$.

Also, given $s \in V$ nonzero, define the order of vanishing $\operatorname{ord}_{D \bullet} s$ along $D \bullet$ to be $\operatorname{deg} D_{i}$, where $i$ is maximal so that $s \in V\left(-D_{i}\right)$.

Thus, multivanishing sequences generalize usual vanishing sequences and ramification, incorporating also geometric notions such as secancy conditions (requiring two or more points to map to a single a point), bitangency, and so forth. Similar conditions for the case of rational curves were studied by García-Puente et al in $\left[\mathrm{GPHH}^{+} 12\right]$. In [Oss14a] we observe that the standard results on Brill-Noether theory with imposed ramification generalize to imposed multivanishing sequences.

Note that due to our choice of indexing of the multivanishing sequence, adding repeated divisors into $D$ • does not affect the sequence.

Now, suppose that $X_{0}$ is obtained by gluing together smooth curves $Z_{1}$ and $Z_{2}$ at nodes $P_{1}, \ldots, P_{m}$. Given $d>0$, let $d_{1}, d_{2}$ be positive integers such that there exists $b \geqslant 0$ with $d=d_{1}+d_{2}-b m$, and suppose also that $d-d_{i}<m$ for $i=1,2$ (in the Eisenbud-Harris case, we will have $d_{1}=d_{2}=b=d$ ). For $i=1,2$ and $0 \leqslant j \leqslant b+1$, set $D_{j}^{i}=j\left(P_{1}+\cdots+P_{m}\right)$. Now, suppose we are given $\left(\mathscr{L}^{i}, V^{i}\right)$ a $\mathfrak{g}_{d_{i}}^{r}$ on $Z_{i}$ for $i=1,2$, and suppose we are also given gluing information $\varphi$ for $\mathscr{L}^{1}$ and $\mathscr{L}^{2}$ at the nodes. Then we define the tuple $\left(\left(\mathscr{L}^{1}, V^{1}\right),\left(\mathscr{L}^{2}, V^{2}\right), \varphi\right)$ to be a limit linear series if the following two conditions are satisfied:

(I) for $i=1,2$, write $a_{0}^{i}, \ldots, a_{r}^{i}$ for the multivanishing sequence of $\left(\mathscr{L}^{i}, V^{i}\right)$ along $D_{\bullet}^{i} ;$ then we require

$$
a_{j}^{1}+a_{r-j}^{2} \geqslant b m
$$

for $j=0, \ldots, r$;

(II) for $i=1,2$, there exist bases $s_{0}^{i}, \ldots, s_{r}^{i}$ of the $V^{i}$ such that

$$
\operatorname{ord}_{D .} s_{\ell}^{i}=a_{\ell}^{i} \quad \text { for } \ell=0, \ldots, r
$$

and for all $\ell$ with (1.2) an equality, we have

$$
\varphi\left(s_{\ell}^{1}\right)=s_{r-\ell}^{2} .
$$

In the above, we have been a bit vague in discussing the gluing; this is made fully precise in $\S 4$ below. Then, in $\S 5$, we generalize to the case of curves of pseudocompact type, meaning that if we take the dual graph, and collapse all multiple edges, we obtain a tree ${ }^{1}$ see Figure 1 . In addition to curves of compact type, this includes interesting classes of curves such as curves with two components, and chains of curves of the sort considered by Cools, Draisma, Payne and Robeva in [CDPR12]. One can think of curves of pseudocompact type as being the most general class of curves for which one can still analyze gluing conditions by looking at only two components at a time. Note that there is a close parallel between the above conditions (I) and (II) and the definition of limit linear series for higher-rank vector bundles given by Teixidor i Bigas in [Tei91]. This parallel persists, albeit to a lesser extent, when we allow insertions of chain of rational curves, and consider arbitrary curves of pseudocompact type. However, this is reflective of the node-by-node aspect of the gluing conditions, and for arbitrary nodal curves the behavior is expected to be quite different.

\footnotetext{
${ }^{1}$ More precisely, we obtain a tree from the dual graph by, for each pair of adjacent vertices $v, v^{\prime}$, replacing all edges connecting $v$ to $v^{\prime}$ with a single edge.
} 


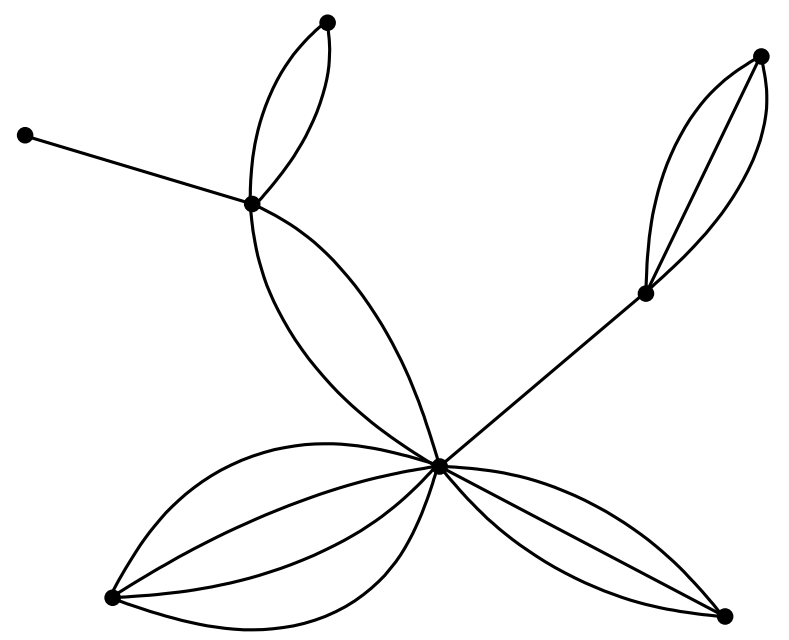

Figure 1. A dual graph of a curve of pseudocompact type.

Finally, in $\S 6$ we prove the following smoothing theorem. We state it informally here, with a more precise statement as Theorem 6.1 below.

Theorem 1.3. If $X_{0}$ is a curve of pseudocompact type, and the space of limit linear on $X_{0}$ has the expected dimension

$$
\rho:=g+(r+1)(d-r-g),
$$

then every limit linear series on $X_{0}$ can be smoothed to linear series on all nearby smooth curves.

As mentioned above, in comparison to the smoothing theorem of EisenbudHarris, our result is stronger because it is not confined to the open subset of refined limit linear series. The main tool in the proof of Theorem 1.3 is the theory of linked determinantal loci, which we develop in Appendix A.

We conclude with a brief explanation of some of the decisions behind our definitions. First, we originally intended to use torsion-free sheaves to treat specializations, rather than allowing the insertion of rational curves at nodes. However, we discovered that from this point of view, important gluing conditions are omitted, and as a result, the spaces may no longer have the correct dimension. Next, of course in a general theory of limit linear series, in principle one does not need to treat inserted rational chains differently from other components. However, there are two compelling reasons for doing so. The first is that it keeps the amount of data more manageable; for instance, in the two-component case, we can study limit linear series in general without having to remember more than two linear series, one for each of the original components. The other reason is that the pseudocompact type condition is not preserved under insertion of rational curves at nodes, so our second definition would not be complete (for instance, with respect to specialization results) if we did not have a system for keeping track of inserted rational curves. In addition, our approach is very convenient for working with non-regular smoothing families. The final comment is that we have not, for the moment, pursued the possibility of creating a single proper moduli space of limit linear series using the quasistable curve compactification of the Picard variety. This is a natural 
and worthwhile direction to pursue, but because Eisenbud and Harris were able to carry out all their applications without a compact moduli space (using instead a specialization result analogous to our Corollary 3.15), it does not seem to be crucial to the basic theory.

Acknowledgements. I would like to thank Eduardo Esteves for many helpful conversations, particularly in relation to chain structures and admissible multidegrees. I would also like to thank Frank Sottile for drawing my attention to [GPHH $\left.{ }^{+} 12\right]$, and Ulrich Goertz for his assistance with the proof of Proposition A.7.

Conventions. All curves we consider are assumed proper, (geometrically) reduced and connected, and at worst nodal. All nodal curves are assumed to be split, meaning that both its nodes and irreducible components are all defined over the base field. Furthermore, to reduce clutter, we assume that all irreducible components are smooth; see Remark 2.23.

A graph by default is allowed to have multiple edges, but not, in accordance with the above, loops.

\section{Fundamental Definitions}

We begin with some definitions of a combinatorial nature. In the below, $\Gamma$ will be obtained by choosing a directed structure on the dual graph of a projective nodal curve. We assume we have:

Situation 2.1. Let $\Gamma$ be a directed graph without loops. For each pair of an edge $e$ and adjacent vertex $v$ of $\Gamma$, let $\sigma(e, v)=1$ if $e$ has tail $v$, and -1 if $e$ has head $v$.

The following definitions form the basis for our approach to keeping track of chains of rational curves inserted at the nodes of the original curve.

Definition 2.2. A chain structure on $\Gamma$ is a function $\boldsymbol{n}: E(\Gamma) \rightarrow \mathbb{Z}_{>0}$. A chain structure is trivial if $\boldsymbol{n}(e)=1$ for all $e \in E(\Gamma)$.

The chain structure will determine the length of the chain of rational curves inserted at a given node; for reasons of later convenience, the trivial case (in which no rational curves are inserted) corresponds to $\boldsymbol{n}(e)=1$.

Definition 2.3. Given $\boldsymbol{n}$ a chain structure on $\Gamma$, an admissible multidegree $w$ of total degree $d$ on $(\Gamma, \boldsymbol{n})$ consists of a function $w_{\Gamma}: V(\Gamma) \rightarrow \mathbb{Z}$ together with a tuple $(\mu(e))_{e \in E(\Gamma)}$, where each $\mu(e) \in \mathbb{Z} / \boldsymbol{n}(e) \mathbb{Z}$, such that

$$
d=\#\{e \in E(\Gamma): \mu(e) \neq 0\}+\sum_{v \in V(\Gamma)} w_{\Gamma}(v) .
$$

The idea behind admissible multidegrees is that in order to extend line bundles, we need only consider multidegrees which have degree 0 or 1 on each rational curve inserted at the node, with degree 1 occurring at most once in each chain. Thus, $\mu(e)$ determines where on the chain (if anywhere) positive degree occurs. See Definition 2.16 below for details.

Definition 2.4. Given a chain structure $\boldsymbol{n}$ on $\Gamma$, let $w$ be an admissible multidegree. Given also $v \in V(\Gamma)$, the twist of $w$ at $v$ is obtained as follows: for each $e$ adjacent to $v$, increase $\mu(e)$ by $\sigma(e, v)$. Now, decrease $w_{\Gamma}(v)$ by the number of $e$ for which $\mu(e)$ had been equal to 0 , and for each $e$, if the new $\mu(e)$ is zero, increase $w_{\Gamma}\left(v^{\prime}\right)$ by 
1 , where $v^{\prime}$ is the other vertex adjacent to $v$. The negative twist of $w$ at $v$ is the admissible multidegree $w^{\prime}$ such that the twist of $w^{\prime}$ at $v$ is equal to $w$.

Twists will be the change in multidegrees accomplished by twisting by certain natural line bundles; see Notation 2.18 below.

Example 2.5. In the case of trivial chain structure, a twist at $v$ simply reduces $w_{\Gamma}(v)$ by the valence of $v$ while increasing $w_{\Gamma}\left(v^{\prime}\right)$ by the number of edges connecting $v^{\prime}$ to $v$, for each $v^{\prime} \neq v$. This is the same as the chip firing considered by Baker and Norine in [BN07].

Remark 2.6. Given $\Gamma$ and $\boldsymbol{n}$, let $\widetilde{\Gamma}$ be the (directed) graph obtained from $\Gamma$ by subdividing each edge $e$ into $\boldsymbol{n}(e)$ edges. Thus, we have a natural inclusion $V(\Gamma) \subseteq$ $V(\widetilde{\Gamma})$. Then if $w$ is an admissible multidegree for $(\Gamma, \boldsymbol{n})$, we obtain a weight function $\widetilde{w}: V(\widetilde{\Gamma}) \rightarrow \mathbb{Z}$ on $\widetilde{\Gamma}$ (which we think of as being a multidegree for the trivial chain structure) by setting $\widetilde{w}(v)=w_{\Gamma}(v)$ for all $v \in V(\Gamma)$, and setting $\widetilde{w}(v)=0$ for all $v \notin V(\Gamma)$, unless $v$ lies over an edge $e$ of $\Gamma$, and is the $\mu(e)$ th new vertex lying over e. In the latter case, we set $\widetilde{w}(v)=1$.

Thus, admissible multidegrees for $(\Gamma, \boldsymbol{n})$ are imbedded into the set of multidegrees on $\widetilde{\Gamma}$, and this imbedding is compatible with twists as follows: twisting $w$ at $v \in V(\Gamma)$ is the same as twisting $\widetilde{w}$ by $v$, and then also by all new vertices between $v$ and the $\sigma(e, v) \mu(e)$ th new vertex lying over $e$, for each $e \in E(\Gamma)$ adjacent to $v$. In the above, we take the representative of $\sigma(e, v) \mu(e)$ between 0 and $\boldsymbol{n}(e)-1$. See also Notation 2.18 below for the geometric version of this statement.

Example 2.7. In the two-component case, with components $v_{1}$ and $v_{2}$, and edges oriented from $v_{1}$ to $v_{2}$, we describe twists in terms of multidegrees on $\widetilde{\Gamma}$ as in Remark 2.6. The idea is that twisting by $v_{1}$ moves the positive-degree new vertices away from $v_{1}$ and towards $v_{2}$. Specifically, when twisting $w$ at $v_{1}$, for each $e \in E(\Gamma)$, the degree- 1 new vertex over $e$ shifts by one away from $v_{1}$. If the vertex with degree 1 is already adjacent to $v_{2}$, then the degree on $v_{2}$ is increased, and no new vertices over $e$ will have positive degree. If no new vertices over $e$ have degree 1 , then the degree on $v_{1}$ is decreased, and the first new vertex over $e$ is given degree 1 .

Note that twists are invertible, since twisting at every vertex of $\Gamma$ returns to the initial multidegree. Thus, the negative twist at $v$ can be expressed also as the composition of the twists at all $v^{\prime} \neq v$. We will primarily be interested in (positive) twists, but the utility for us of negative twists is in the following definition.

Definition 2.8. An admissible multidegree $w$ is concentrated at a vertex $v \in$ $V(\Gamma)$ if there is an ordering on $V(\Gamma)$ starting with $v$, and such that for each subsequent vertex $v^{\prime}$, we have that $w$ becomes negative in index $v^{\prime}$ after taking the composition of the negative twists at all the previous vertices.

A more canonical condition which implies concentration (but is in general strictly stronger) is that for all $v^{\prime} \neq v$, and all $v^{\prime \prime}$ adjacent to $v^{\prime}$, the negative twist of $w$ at $v^{\prime \prime}$ is negative in index $v^{\prime}$. We have elected to use the above definition as the most general for which one can make the argument of Proposition 3.3 below.

Example 2.9. The concentration condition is the generalization of the multidegrees considered by Eisenbud and Harris in the compact type case, where they had degree $d$ on one component, and degree 0 on all the others. 
In our generalized setting, $w$ will be concentrated at $v$ if it is negative on all $v^{\prime} \neq v$. If the chain structure is trivial, it is enough to have degree at most 0 at all $v^{\prime} \neq v$, but in general this is not the case, since with nontrivial chain structures, a negative twist at $v^{\prime \prime}$ adjacent to $v^{\prime}$ need not reduce the degree on $v^{\prime}$.

However, at the opposite extreme, even with nontrivial chain structures we can have a multidegree simultaneously concentrated at two adjacent vertices. For instance, if $\Gamma$ has only two vertices, connected by $n$ edges, then a multidegree which is strictly less than $n$ on each vertex, and with $\mu(\bullet)$ identically zero, will be concentrated on both vertices. This corresponds to usual linear series (of restricted multidegrees) on the relevant two-component curves.

See also Remark 2.24 below for further comments on the role of the concentration condition.

Proposition 2.10. Given any admissible multidegree $w$, and any $v \in V(\Gamma)$, there exists an admissible multidegree $w^{\prime}$, concentrated at $v$, and obtained from $w$ by repeated twisting at vertices $v^{\prime}$ other than $v$.

Proof. First note that the composition of negative twists over a collection $S$ of vertices of $\Gamma$ is equivalent to the composition of (positive) twists over the complement of $S$. For each $n \geqslant 0$, let $\Gamma_{v, n}$ denote the subset of $V(\Gamma)$ consisting of all vertices $v^{\prime}$ such that there is a path (undirected) in $\Gamma$ of length less than or equal to $n$ from $v$ to $v^{\prime}$. Let $N$ be maximal such that $\Gamma_{v, N} \subsetneq V(\Gamma)$. Taking sufficiently many negative twists of $w$ at all vertices of $\Gamma_{v, N}$, we can achieve negative degrees at all vertices of $V(\Gamma) \backslash \Gamma_{v, N}$. Repeating this process for $\Gamma_{v, N-1}$ achieves negative degree on $\Gamma_{v, N} \backslash \Gamma_{v, N-1}$ without affecting the degree on $V(\Gamma) \backslash \Gamma_{v, N}$, and continuing in this way down to $\Gamma_{v, 0}$, we achieve negative degree at all vertices other than $v$, which in particular implies concentration at $v$.

The following directed graph keeps track of all the multidegrees we will want to consider starting from any one admissible multidegree.

Notation 2.11. Let $G\left(w_{0}\right)$ be the directed graph with vertex set

$$
V\left(G\left(w_{0}\right)\right) \subseteq \mathbb{Z}^{V(\Gamma)} \times \prod_{e \in E(\Gamma)} \mathbb{Z} / \boldsymbol{n}(e) \mathbb{Z}
$$

consisting of all admissible multidegrees obtained from $w_{0}$ by sequences of twists, and with an edge from $w$ to $w^{\prime}$ if $w^{\prime}$ is obtained from $w$ by twisting at some vertex $v$ of $\Gamma$.

Given $w \in V\left(G\left(w_{0}\right)\right)$ and $v_{1}, \ldots, v_{m} \in V(\Gamma)$ (not necessarily distinct), let $P\left(w, v_{1}, \ldots, v_{m}\right)$ denote the path in $V\left(G\left(w_{0}\right)\right)$ obtained by starting at $w$, and twisting successively at each $v_{i}$.

By the invertibility of twists, $G\left(w_{0}\right)=G(w)$ if and only if $w \in G\left(w_{0}\right)$. While our directed structure on $\Gamma$ is just a convenience, the directedness of $G\left(w_{0}\right)$ is crucial. Although it is not important for our present purposes, we also mention that $G\left(w_{0}\right)$ can be expressed as the collection of admissible multidegrees which are linearly equivalent to $w_{0}$ on $\widetilde{\Gamma}_{0}$, using the theory of linear equivalence on graphs as developed by Baker and Norine in [BN07].

Also, note that $P\left(w, v_{1}, \ldots, v_{m}\right)$ is independent of the ordering of the $v_{i}$.

Proposition 2.12. If $P\left(w, v_{1}, \ldots, v_{m}\right)$ is a minimal path in $G\left(w_{0}\right)$ from $w$ to some $w^{\prime}$, then $m$ and the $v_{i}$ are uniquely determined up to reordering. 
More generally, paths $P\left(w, v_{1}, \ldots, v_{m}\right)$ and $P\left(w, v_{1}^{\prime}, \ldots, v_{m^{\prime}}^{\prime}\right)$ have the same endpoint if and only if the multisets of the $v_{i}$ and the $v_{i}^{\prime}$ differ by a multiple of $V(\Gamma)$.

Proof. We have already observed the "if" direction. For the converse, in light of Remark 2.6 the desired statement for $\Gamma$ and $\boldsymbol{n}$ follows from the same statement for the graph $\widetilde{\Gamma}$ constructed by subdividing every edge $e$ of $\Gamma$ into $\boldsymbol{n}(e)$ edges, with the trivial chain structure. We thus consider the matrix $M$ indexed by $V(\widetilde{\Gamma})$, with $(v, v)$ entry given by the negative of the valence of $v$, and for $v \neq v^{\prime}$, with $\left(v, v^{\prime}\right)$ entry given by the number of edges of $\widetilde{\Gamma}$ connecting $v$ to $v^{\prime}$. We wish to see that the vector $(1, \ldots, 1)$ generates the kernel of $M$. If we consider $\epsilon M+I$, with $1 / \epsilon$ at least the maximal valence in $\widetilde{\Gamma}$, we have a symmetric doubly stochastic matrix with nonnegative entries, which is irreducible because $\Gamma$ is connected. The PerronFrobenius theorem then implies that the maximal eigenvalue is 1 , and is simple, which implies that the eigenvalue 0 of $M$ is likewise simple, as desired.

We now move on to definitions which involve geometry more directly.

Situation 2.13. Let $X_{0}$ be a projective nodal curve, with dual graph $\Gamma$, and choose an orientation on $\Gamma$. For $v \in V(\Gamma)$, let $Z_{v}$ be the corresponding irreducible component of $X_{0}$, and $Z_{v}^{c}$ the closure of the complement of $Z_{v}$ in $X_{0}$.

A preliminary definition (see also Maino [Mai98]) is the following.

Definition 2.14. If $X_{0}$ is a nodal curve with dual graph $\Gamma$, an enriched structure on $X_{0}$ consists of the data, for each $v \in V(\Gamma)$ of a line bundle $\mathscr{O}_{v}$ on $X_{0}$, satisfying the following conditions:

(I) for any $v \in V(\Gamma)$, we have

$$
\left.\mathscr{O}_{v}\right|_{Z_{v}} \cong \mathscr{O}_{Z_{v}}\left(-\left(Z_{v}^{c} \cap Z_{v}\right)\right) \text {, and }\left.\mathscr{O}_{v}\right|_{Z_{v}^{c}} \cong \mathscr{O}_{Z_{v}^{c}}\left(Z_{v}^{c} \cap Z_{v}\right)
$$

(II) we have

$$
\bigotimes_{v \in V(\Gamma)} \mathscr{O}_{v} \cong \mathscr{O}_{X_{0}}
$$

Note that it follows from the definitions that each $\mathscr{O}_{v}$ has degree 0 . Enriched structures always exist; they amount to suitable gluing choices at the nodes, and they are unique when $X_{0}$ is of compact type. However, an enriched structure is always induced by any regular smoothing of $X_{0}$; see Proposition 3.10.

We now explicitly introduce the chains of rational curves induced by a chain structure on $X_{0}$.

Definition 2.15. Given $X_{0}$ and a chain structure $\boldsymbol{n}$, let $\widetilde{X}_{0}$ denote the nodal curve obtained from $X_{0}$ by, for each $e \in E(\Gamma)$, inserting a chain of $\boldsymbol{n}(e)-1$ projective lines at the corresponding node. Let $\widetilde{\Gamma}$ be the dual graph of $\tilde{X}_{0}$, with a natural inclusion $V(\Gamma) \subseteq V(\widetilde{\Gamma})$. We refer to the new components of $\widetilde{X}_{0}$ as the exceptional components.

Note that the above $\widetilde{\Gamma}$ is compatible with that of Remark 2.6.

Definition 2.16. Using our orientation of $E(\Gamma)$, an admissible multidegree $w$ of total degree $d$ on $\left(X_{0}, \boldsymbol{n}\right)$ gives a multidegree of total degree $d$ on $\widetilde{X}_{0}$ by assigning, for each $e \in E(\Gamma)$, degree 0 on each component of the corresponding chain of projective curves, except for degree 1 on the $\mu(e)$ th component when $\mu(e) \neq 0$. 
The reason for restricting to such multidegrees is that extensions of line bundles may always be chosen to have such degrees; see Corollary 3.15.

From now on, we will assume we have fixed an enriched structure together with suitable global sections, as follows.

Situation 2.17. In Situation 2.13, suppose we have also a chain structure $\boldsymbol{n}$ on $\Gamma$, and an enriched structure $\left(\mathscr{O}_{v}\right)_{v}$ on the resulting $\widetilde{X}_{0}$, and for each $v \in V(\widetilde{\Gamma})$, fix $s_{v} \in \Gamma\left(\tilde{X}_{0}, \mathscr{O}_{v}\right)$ vanishing precisely on $Z_{v}$.

The sections $s_{v}$ will be convenient in describing maps between different twists of line bundles; they will not be unique even for curves of compact type, but in our case they are just a formal convenience, and do not ultimately affect our definition of limit linear series. See Remark 2.22 for further discussion.

We next describe how, given an enriched structure on $\widetilde{X}_{0}$, and a line bundle $\mathscr{L}$ of multidegree $w_{0}$, we get a collection of line bundles indexed by $V\left(G\left(w_{0}\right)\right)$, with morphisms between them indexed by $E\left(G\left(w_{0}\right)\right)$.

Notation 2.18. In Situation 2.17 assume we are given also an admissible multidegree $w_{0}$ on $(\Gamma, \boldsymbol{n})$. Then for any edge $\varepsilon \in E\left(G\left(w_{0}\right)\right)$, starting at $w=\left(w_{\Gamma},(\mu(e))_{e \in E(\Gamma)}\right)$ and determined by twisting at $v \in V(\Gamma)$, we have the corresponding twisting line bundle $\mathscr{O}_{\varepsilon}$ on $\tilde{X}_{0}$ defined as

$$
\mathscr{O}_{\varepsilon}=\mathscr{O}_{v} \otimes \bigotimes_{e \in E(\Gamma)} \bigotimes_{i=1}^{\sigma(e, v) \mu(e)} \mathscr{O}_{v_{e, i}},
$$

where the first product is over edges $e$ adjacent to $v$, and for any such pair, $v_{e, i}$ denotes the $i$ th rational curve in $\widetilde{X}_{0}$ from $Z_{v}$ on the chain corresponding to $e$.

In addition, we have the section $s_{\varepsilon}$ of $\mathscr{O}_{\varepsilon}$ obtained from the tensor product of the relevant sections $s_{v}$ and $s_{v_{e, i}}$.

Similarly, given $w, w^{\prime} \in V\left(G\left(w_{0}\right)\right)$, let $P=\left(\varepsilon_{1}, \ldots, \varepsilon_{m}\right)$ be a minimal path from $w$ to $w^{\prime}$ in $G\left(w_{0}\right)$, and set

$$
\mathscr{O}_{w, w^{\prime}}=\bigotimes_{i=1}^{m} \mathscr{O}_{\varepsilon_{i}}
$$

In Notation 2.18, if $\mu(e)=0$, the product over $i$ is empty for the given $e$, and we take the representative of $\sigma(e, v) \mu(e)$ between 0 and $\boldsymbol{n}(e)-1$. Note that it follows from Proposition 2.12 that the constructions of Notation 2.18 are independent of choices of (minimal) paths. The reason for the notation $\mathscr{O}_{w, w^{\prime}}$ is that, as one can easily verify, tensoring by $\mathscr{O}_{w, w^{\prime}}$ take a line bundle of multidegree $w$ to one of multidegree $w^{\prime}$.

Notation 2.19. In Situation 2.17, suppose $\mathscr{L}$ is a line bundle on $\tilde{X}_{0}$ of multidegree $w_{0}$. Then for any $w \in V\left(G\left(w_{0}\right)\right)$, set

$$
\mathscr{L}_{w}:=\mathscr{L} \otimes \mathscr{O}_{w_{0}, w} .
$$

Given also $w_{v} \in V\left(G\left(w_{0}\right)\right)$ concentrated at $v$, set

$$
\mathscr{L}^{v}:=\left.\mathscr{L}_{w_{v}}\right|_{Z_{v}} .
$$

Given an edge $\varepsilon$ from $w$ to $w^{\prime}$ in $G\left(w_{0}\right)$, corresponding to twisting at $v$, then either $\mathscr{L}_{w^{\prime}}=\mathscr{L}_{w} \otimes \mathscr{O}_{\varepsilon}$, or $\mathscr{L}_{w}=\mathscr{L}_{w^{\prime}} \otimes \mathscr{O}_{w^{\prime}, w}$. In the former case, we get a morphism 
$\mathscr{L}_{w} \rightarrow \mathscr{L}_{w^{\prime}}$ induced by $s_{\varepsilon}$. In the latter case, we observe that $\mathscr{O}_{w^{\prime}, w} \otimes \mathscr{O}_{\varepsilon} \cong \mathscr{O}_{\widetilde{X}_{0}}$, and fixing such an isomorphism and again using $s_{\varepsilon}$ gives an induced morphism

$$
\mathscr{L}_{w} \rightarrow \mathscr{L}_{w} \otimes \mathscr{O}_{\varepsilon}=\mathscr{L}_{w^{\prime}} \otimes \mathscr{O}_{w^{\prime}, w} \otimes \mathscr{O}_{\varepsilon} \cong \mathscr{L}_{w^{\prime}}
$$

In either case, pushing forward gives an induced morphism

$$
f_{\varepsilon}: \Gamma\left(\tilde{X}_{0}, \mathscr{L}_{w}\right) \rightarrow \Gamma\left(\tilde{X}_{0}, \mathscr{L}_{w^{\prime}}\right)
$$

Finally, if $P=\left(\varepsilon_{1}, \ldots, \varepsilon_{m}\right)$ is any path in $G\left(w_{0}\right)$, set

$$
f_{P}:=f_{\varepsilon_{m}} \circ \cdots \circ f_{\varepsilon_{1}} .
$$

If $P$ is a minimal path from $w$ to $w^{\prime}$, write

$$
f_{w, w^{\prime}}:=f_{P}
$$

We have the following simple consequence of Proposition 2.12:

Corollary 2.20. For any $w, w^{\prime} \in V\left(G\left(w_{0}\right)\right)$, the morphism $f_{w, w^{\prime}}$ is independent of the choice of minimal path.

We can now give the definition of a limit linear series. As mentioned previously, the idea is simply that a collection of $\mathfrak{g}_{d_{v}}^{r}$ s on the components $Z_{v}$ of $X_{0}$ should constitute a limit linear series precisely when it is possible to use them to glue together an $(r+1)$-dimensional space of sections on all of $X_{0}$ in any desired multidegree.

Definition 2.21. Let $X_{0}$ be a projective nodal curve, $\boldsymbol{n}$ a chain structure, $w_{0}$ an admissible multidegree of total degree $d$ on $\left(X_{0}, \boldsymbol{n}\right)$, and $\left(\mathscr{O}_{v}\right)_{v \in V(\Gamma)}$ an enriched structure on $\tilde{X}_{0}$. Choose also a tuple $\left(w_{v}\right)_{v \in V(\Gamma)}$ of vertices of $G\left(w_{0}\right)$, with each $w_{v}$ concentrated at $v$, and sections $\left(s_{v}\right)_{v}$ as in Situation 2.17. Then a limit linear series on $\left(X_{0}, \boldsymbol{n}\right)$ consists of a line bundle $\mathscr{L}$ of multidegree $w_{0}$ on $\tilde{X}_{0}$, together with subspaces $V^{v}$ of $\Gamma\left(Z_{v}, \mathscr{L}^{v}\right)$ for each $v \in V(\Gamma)$, satisfying the condition that for all $w \in V\left(G\left(w_{0}\right)\right)$, the natural morphism

$$
\Gamma\left(\tilde{X}_{0}, \mathscr{L}_{w}\right) \rightarrow \bigoplus_{v \in V(\Gamma)} \Gamma\left(Z_{v}, \mathscr{L}^{v}\right) / V^{v}
$$

has kernel of dimension at least $r+1$, where (2.1) is obtained as the composition

$$
\begin{aligned}
\Gamma\left(\widetilde{X}_{0}, \mathscr{L}_{w}\right) \stackrel{\oplus f_{w_{w} w_{v}}}{\rightarrow} \bigoplus_{v \in V(\Gamma)} \Gamma\left(\widetilde{X}_{0}, \mathscr{L}_{w_{v}}\right) & \\
& \rightarrow \bigoplus_{v \in V(\Gamma)} \Gamma\left(Z_{v}, \mathscr{L}^{v}\right) \rightarrow \bigoplus_{v \in V(\Gamma)} \Gamma\left(Z_{v}, \mathscr{L}^{v}\right) / V^{v} .
\end{aligned}
$$

Clearly, the choices of concentrated multidegrees are necessary to even define the data underlying a limit linear series. However, we will show in Proposition 3.5 below that the resulting moduli space of limit linear series does not depend on this choice.

Remark 2.22. Even in the compact type case, the sections $s_{v}$ of Situation 2.17 are not typically unique, even up to scaling: indeed, if $v$ disconnects $\Gamma$, then $s_{v}$ can be scaled independently on (the subcurves corresponding to) each resulting connected component. Thus, a priori our definition of limit linear series depends on extra data even in the compact type case. However, the choice of $s_{v}$ is unique up to scaling on each component, and because the maps (2.1) are obtained by restricting to individual components, their kernels do not depend on the choice of 
$s_{v}$. Consequently, we see that the notion of limit linear series is in fact independent of the choices of the $s_{v}$. This is different from the notion of linked linear series introduced in [Oss14c] (generalizing [Oss06]), where even for curves of compact type, the choice of $s_{v}$ does have an effect.

Remark 2.23. We have chosen not to allow self-nodes (i.e., nodes on single irreducible components) not because they are harder to handle, but because they are already better understood, and our techniques don't add anything new for them. It is not difficult to combine our techniques with those developed for self-nodes, but we have chosen to present our definitions and results without any self-nodes because we would have to systematically treat the two types of nodes differently.

If one wants to treat limit linear series on a reducible curve with some self-nodes, there are several options: the first, which is simplest to state but probably least effective for computation is to simply introduce new rational components at each self-node, thereby removing all self-nodes; another option is to work with sheaves which are allowed to be torsion-free (but not invertible) at the self-nodes. In the latter case, one can study the resulting linear series by partially normalizing at the self-nodes and studying linear series on the resulting smooth component(s), imposing a secancy condition at each pair of points lying above self-nodes at which the sheaf was invertible (above nodes at which the sheaf was not invertible, one does not have a gluing condition, but the degree on the relevant component is decreased). This approach was developed already by Kleiman [Kle76] nearly 40 years ago.

Remark 2.24. Obviously, concentrated multidegrees are not unique, so a choice of these is a necessary input to our definition of limit linear series. Although Proposition 3.5 asserts that in fact the resulting limit linear series moduli spaces will not depend on the choice of the $w_{v}$, it is still natural to wonder to what extent one can make canonical choices of the tuples $\left(w_{v}\right)_{v}$ of concentrated multidegrees. The answer likely comes from the theory of $v$-reduced divisors, which plays an important role in Brill-Noether theory for graphs. However, since our theory goes through fully as long as the $w_{v}$ are concentrated, it seems potentially advantageous not to place any further restrictions on them. Thus, the question of canonical choices is rather orthogonal to the purpose of the present paper, and for the sake of simplicity we do not pursue it here.

\section{FAMilies AND MODULI SCHEMES}

In this section, we construct a moduli scheme of limit linear series, show that it is independent of the choice of tuple of concentrated multidegree, and finally give an alternate description which generalizes to the case of smoothing families. The main technical tool is the generalized determinantal loci introduced in Appendix B of [Oss14c], and the main issue that needs to be addressed for smoothing families is that the limit linear series are defined in terms of (sections of) line bundles $\mathscr{L}^{v}$ on individual components of the reducible curve, which no longer makes sense in a smoothing family. This difficulty is resolved by working instead with the line bundles $\mathscr{L}_{w_{v}}$ on the whole curve, with multidegree concentrated on the relevant component.

This section is of a foundational nature, and later sections are largely independent from it, with the exception of Theorem 6.1, our smoothing result. Note, however, that our specialization result, Corollary 3.15 , is proved in this section. 
First, we set the following notation.

Notation 3.1. In the situation of Definition 2.21, let $P_{w_{\bullet}}^{r}\left(X_{0}, \boldsymbol{n},\left(\mathscr{O}_{v}\right)_{v}\right)$ be the scheme parametrizing tuples $\left(\mathscr{L},\left(V^{v}\right)_{v \in V(\Gamma)}\right)$, where $\mathscr{L}$ is a line bundle on $\tilde{X}_{0}$ of multidegree $w_{0}$, and each $V^{v}$ is an $(r+1)$-dimensional space of global sections of the induced line bundle $\mathscr{L}^{v}$ on $Z_{v}$.

Thus, if we write $d_{v}=\operatorname{deg} \mathscr{L}^{v}$, then $P_{w_{\bullet}}^{r}\left(X_{0}, \boldsymbol{n},\left(\mathscr{O}_{v}\right)_{v}\right)$ can naturally be constructed as a fibered product of $\operatorname{Pic}^{w_{0}}\left(\widetilde{X}_{0}\right)$ with the spaces $G_{d_{v}}^{r}\left(Z_{v}\right)$, fibered over the spaces $\operatorname{Pic}^{d_{v}}\left(Z_{v}\right)$.

We then construct a moduli scheme of limit linear series as follows.

Definition 3.2. In the situation of Definition 2.21, write $\mathscr{M}$ for the universal line bundle on $P_{w_{\bullet}}^{r}\left(X_{0}, \boldsymbol{n},\left(\mathscr{O}_{v}\right)_{v}\right) \times X_{0}$, and $\mathscr{V}^{v}$ for the universal subbundles of the induced $p_{1 *} \mathscr{M}^{v}$. Then let $G_{\bar{w}_{0}}^{r}\left(X_{0}, \boldsymbol{n},\left(\mathscr{O}_{v}\right)_{v}\right)$ be the closed subscheme of $P_{w_{\bullet}}^{r}\left(X_{0}, \boldsymbol{n},\left(\mathscr{O}_{v}\right)_{v}\right)$ defined by the intersection over $w \in V\left(G\left(w_{0}\right)\right)$ of the $(r+1)$ st vanishing loci of the maps

$$
p_{1 *} \mathscr{M}_{w} \rightarrow \bigoplus_{v \in V(\Gamma)}\left(p_{1 *} \mathscr{M}^{v}\right) / \mathscr{V}^{v}
$$

In the above, the $(r+1)$ st vanishing locus is a canonical scheme structure on the set of points on which the kernel has dimension at least $r+1$, defined in Appendix B of [Oss14c]. Thus, $G_{\bar{w}_{0}}^{r}\left(X_{0}, \boldsymbol{n},\left(\mathscr{O}_{v}\right)_{v}\right)$ is a canonical scheme structure on the set of limit linear series described in Definition 2.21. The notation $\bar{w}_{0}$ represents the collection of admissible multidegrees obtained from $w_{0}$ by twisting (that is, $\left.V\left(G\left(w_{0}\right)\right)\right)$; we use it because we will prove shortly, in Proposition 3.5, that the choice of the $w_{v}$ does not affect the resulting moduli scheme.

The $T$-valued points of $P_{w_{\bullet}}^{r}\left(X_{0}, \boldsymbol{n},\left(\mathscr{O}_{v}\right)_{v}\right)$ are tuples $\left(\mathscr{L},\left(V^{v}\right)\right)$, where $\mathscr{L}$ is a line bundle on $T \times \tilde{X}_{0}$ of multidegree $w_{0}$, and each $V^{v}$ is a rank- $(r+1)$ subbundle of $p_{1 *} \mathscr{L}^{v}$ (in the sense of Definition 4.2 of [Oss06]). Such a tuple is a $T$-valued point of $G_{\bar{w}_{0}}^{r}\left(X_{0}, \boldsymbol{n},\left(\mathscr{O}_{v}\right)_{v}\right)$ if for all $w \in V\left(G\left(w_{0}\right)\right)$, the map

$$
p_{1 *} \mathscr{L}_{w} \rightarrow \bigoplus_{v \in V(\Gamma)}\left(p_{1 *} \mathscr{L}^{v}\right) / V^{v}
$$

has $(r+1)$ st vanishing locus equal to all of $T$.

Our next task is to show that in fact, for a fixed $w_{0}$, the spaces of limit linear series for different choices of the $w_{v}$ are canonically identified with one another.

A preliminary fact is the following.

Proposition 3.3. Let $\mathscr{L}$ be a line bundle of multidegree $w \in V\left(G\left(w_{0}\right)\right)$ on $\tilde{X}_{0}$, and suppose that $w$ is concentrated at $v$. Then the restriction map

$$
H^{0}\left(\tilde{X}_{0}, \mathscr{L}\right) \rightarrow H^{0}\left(Z_{v},\left.\mathscr{L}\right|_{Z_{v}}\right)
$$

is injective.

Proof. The main point is that for any vertices $v^{\prime}, v^{\prime \prime}$, and any section $s \in H^{0}\left(\tilde{X}_{0}, \mathscr{L}\right)$ which vanishes on $Z_{v^{\prime \prime}}$, then the number of zeroes consequently imposed on $Z_{v^{\prime}}$ is equal to the change in index $v^{\prime}$ when we take the negative twist of $w$ at $v^{\prime \prime}$. Indeed, if there are $m$ nodes of $X_{0}$ connecting $Z_{v^{\prime}}$ to $Z_{v^{\prime \prime}}$ for which $\mathscr{L}$ is trivial on the associated exceptional chain (equivalently, for which $\mu(e)=0$ ), then $\left.s\right|_{Z_{v^{\prime}}}$ must vanish at these $m$ nodes, but $m$ is also the amount by which the negative twist of 
$w$ at $v^{\prime \prime}$ reduces the degree at $v^{\prime}$. Given this, if $s$ vanishes on $v$, then we simply traverse $\Gamma$ in the ordering provided by the definition of concentration, and vanishing on $Z_{v^{\prime}}$ for all the previous vertices $v^{\prime}$ implies vanishing at the next component as well.

Corollary 3.4. Suppose that $w$ is concentrated at $v$, that $w_{v}$ can be obtained from $w$ by twisting at vertices other than $v$, and that we have a $T$-valued tuple $\left(\mathscr{L},\left(V^{v}\right)_{v}\right)$ such that the $(r+1)$ st vanishing locus of $(3.2)$ is all of $T$. Then the kernel of (3.2) is a subbundle of $p_{1 *} \mathscr{L}_{w}$ of rank $r+1$, and is equal to the preimage of $V^{v}$ under the map $p_{1 *} \mathscr{L}_{w} \rightarrow p_{1 *} \mathscr{L}^{v}$. Moreover, both statements hold after arbitrary base change.

Proof. First observe that the hypotheses on $w$, together with Proposition 3.3, imply that the map

$$
\left.p_{1 *} \mathscr{L}_{w} \rightarrow p_{1 *} \mathscr{L}_{w}\right|_{Z_{v}} \rightarrow p_{1 *} \mathscr{L}^{v}
$$

is injective on points, and hence universally injective. Now, by hypothesis the $(r+1)$ st vanishing locus of $(3.2)$ is all of $T$. On the other hand, at any point, the kernel is contained in the preimage of (the corresponding fiber of) $V^{v}$, which has dimension $r+1$ by the above injectivity. Thus, the $(r+2)$ nd vanishing locus of (3.2) is empty. The statement of the corollary then follows from Proposition B.3.4 and Lemma B.2.3 (iv) of [Oss14c].

Proposition 3.5. In the situation of Definition 2.21, let $\left(w_{v}^{\prime}\right)_{v}$ be another choice of admissible multidegrees concentrated at the $v \in V(\Gamma)$. Then the schemes $G_{\bar{w}_{0}}^{r}\left(X_{0}, \boldsymbol{n},\left(\mathscr{O}_{v}\right)_{v}\right)$ obtained from $\left(w_{v}\right)_{v}$ and $\left(w_{v}^{\prime}\right)_{v}$ are canonically isomorphic.

Proof. It is clearly enough to treat the case that $w_{v^{\prime}}^{\prime}=w_{v^{\prime}}$ for all $v^{\prime}$ other than some fixed choice of $v$. We first observe that it follows from Proposition 2.12 that given any $w, w^{\prime} \in V\left(G\left(w_{0}\right)\right)$, there is some $w^{\prime \prime}$ such that the minimal paths from $w$ to $w^{\prime \prime}$ and from $w^{\prime}$ to $w^{\prime \prime}$ do not require twisting at $v$. Indeed, if we take a minimal path $P$ from $w$ to $w^{\prime}$, and let $w^{\prime \prime}$ be obtained by all twists in $P$ except those at $v$, then the minimal path from $w^{\prime \prime}$ to $w^{\prime}$ involves only twists at $v$, so the minimal path from $w^{\prime}$ to $w^{\prime \prime}$ does not require twisting at $v$. Moreover, if $w^{\prime}$ is concentrated at $v$, then we see from the construction that $w^{\prime \prime}$ is also concentrated at $v$. Thus, to prove the proposition we may further assume that $w_{v}^{\prime}$ is obtained from $w_{v}$ by twisting at vertices other than $v$. In particular, if, for a given $\mathscr{L}$ of multidegree $w_{0}$, we let $\mathscr{L}^{v}$ be as usual, and $\mathscr{L}^{\prime v}$ the corresponding line bundle obtained from $w_{v}^{\prime}$, then the map $\mathscr{L}^{v} \rightarrow \mathscr{L}^{\prime v}$ is (universally) injective. It follows that a subbundle $V^{v}$ of $\mathscr{L}^{v}$ induces a subbundle $V^{\prime v}$ of $\mathscr{L}^{\prime v}$, so we obtain a morphism

$$
P_{w_{\bullet}}^{r}\left(X_{0}, \boldsymbol{n},\left(\mathscr{O}_{v}\right)_{v}\right) \rightarrow P_{w_{\bullet}^{\prime}}^{r}\left(X_{0}, \boldsymbol{n},\left(\mathscr{O}_{v}\right)_{v}\right)
$$

which we wish to show is an isomorphism on the closed subschemes of limit linear series.

First, if $\left(\mathscr{L},\left(V^{v}\right)_{v}\right)$ is a $T$-valued point of $G_{\bar{w}_{0}}^{r}\left(X_{0}, \boldsymbol{n},\left(\mathscr{O}_{v}\right)_{v}\right) \subseteq P_{w_{\bullet}}^{r}\left(X_{0}, \boldsymbol{n},\left(\mathscr{O}_{v}\right)_{v}\right)$, and $w \in V\left(G\left(w_{0}\right)\right)$, we need to check that the $(r+1)$ st vanishing locus of

$$
p_{1 *} \mathscr{L}_{w} \rightarrow p_{1 *} \mathscr{L}^{\prime v} / V^{\prime v} \oplus \underset{v^{\prime} \neq v}{\bigoplus}\left(p_{1 *} \mathscr{L}^{v^{\prime}}\right) / V^{v^{\prime}}
$$

is all of $T$. But by construction, $p_{1 *} \mathscr{L}^{v} / V^{v}$ injects into $p_{1 *} \mathscr{L}^{\prime v} / V^{\prime v}$ (universally), so if there is a minimal path from $w$ to $w_{v}^{\prime}$ factoring through $w_{v}$, then the kernel of (3.3) is identified with that of (3.2), so the hypothesis that $\left(\mathscr{L},\left(V^{v}\right)_{v}\right)$ is in $G_{\bar{w}_{0}}^{r}\left(X_{0}, \boldsymbol{n},\left(\mathscr{O}_{v}\right)_{v}\right)$ together with Proposition B.3.2 of [Oss14c] implies that the $(r+$ 
1)st vanishing locus of (3.3) is all of $T$. Otherwise, we have that the composition of minimal paths from $w$ to $w_{v}$ and from $w_{v}$ to $w_{v}^{\prime}$ is not minimal, meaning that it includes a twist at $v$; since the latter does not have such a twist, we conclude that the minimal path from $w$ to $w_{v}$ includes a twist at $v$. In this case, the minimal path from $w_{v}$ to $w$ does not include a twist at $v$. Let $V_{v}$ denote the kernel of (3.2) in multidegree $w_{v}$; by Corollary 3.4 this is a subbundle which is equal to the preimage of $V^{v}$. Because the minimal path from $w_{v}$ to $w$ does not include a twist at $v$, and $w_{v}$ is concentrated at $v$, we see that the map $p_{1 *} \mathscr{L}_{w_{v}} \rightarrow p_{1 *} \mathscr{L}_{w}$ is universally injective, so the image of $V_{v}$ is a subbundle of rank $r+1$, which is easily verified to be in the kernel of (3.3), since $V_{v}$ is in the kernel of (3.2). We conclude from Proposition B.3.4 of [Oss14c] that the $(r+1)$ st vanishing locus is all of $T$, as desired.

Now, suppose that $\left(\mathscr{L},\left(V^{\prime v}\right)_{v}\right)$ is a $T$-valued point of $G_{\bar{w}_{0}}^{r}\left(X_{0}, \boldsymbol{n},\left(\mathscr{O}_{v}\right)_{v}\right) \subseteq$ $P_{w_{\bullet}^{\prime}}^{r}\left(X_{0}, \boldsymbol{n},\left(\mathscr{O}_{v}\right)_{v}\right)$. In order to lift to $P_{w_{\bullet}}^{r}\left(X_{0}, \boldsymbol{n},\left(\mathscr{O}_{v}\right)_{v}\right)$, we will set $V^{v^{\prime}}=V^{\prime v^{\prime}}$ for all $v^{\prime} \neq v$. At $v$, we consider (3.3) for $w=w_{v}$, and apply Corollary 3.4 again to conclude that the kernel of (3.3) is a subbundle of rank $r+1$ which is equal to the preimage of $V^{\prime v}$ under the universal injection

$$
p_{1 *} \mathscr{L}_{w_{v}} \hookrightarrow p_{1 *} \mathscr{L}^{v} \hookrightarrow p_{1 *} \mathscr{L}^{\prime v}
$$

Put differently, $V^{\prime v}$ must be contained in (the image of) $p_{1 *} \mathscr{L}_{w_{v}}$. Then set $V^{v}$ to be the preimage of $V^{v}$ in $p_{1 *} \mathscr{L}^{v}$, or equivalently, the image of the kernel of (3.3). This gives a $\left(T\right.$-valued) point of $P_{w_{\bullet}}^{r}\left(X_{0}, \boldsymbol{n},\left(\mathscr{O}_{v}\right)_{v}\right)$ mapping to $\left(\mathscr{L},\left(V^{\prime v}\right)_{v}\right)$, and it is clear from the above injectivities that such a point is unique. It thus remains to check that the point we have constructed lies in $G_{\bar{w}_{0}}^{r}\left(X_{0}, \boldsymbol{n},\left(\mathscr{O}_{v}\right)_{v}\right)$.

Given any $w$, we know that the kernel of (3.3) has $(r+1)$ st vanishing locus equal to $T$, and we wish to verify the same for the kernel of (3.2). If there is a minimal path from $w$ to $w_{v}^{\prime}$ factoring through $w_{v}$, then we are in the same situation as above, and we get the desired statement. On the other hand, if the minimal path from $w$ to $w_{v}$ includes a twist at $v$, then in (3.2) the map to the summand $\left(p_{1 *} \mathscr{L}^{v}\right) / V^{v}$ is zero, so we conclude that (3.2) factors through (3.3), and then by Corollary B.3.5 of [Oss14c] it follows that the $(r+1)$ st vanishing locus of $(3.2)$ is all of $T$. The proposition follows.

We now describe a second version of the moduli space construction, which is less immediately related to our definition of limit linear series, but which works transparently in families of curves; we will then show in Proposition 3.8 that on the special fiber, the two constructions are canonically isomorphic.

Notation 3.6. In the situation of Definition 2.21, let $\widetilde{P}_{w \bullet}^{r}\left(X_{0}, \boldsymbol{n},\left(\mathscr{O}_{v}\right)_{v}\right)$ be the scheme parametrizing tuples $\left(\mathscr{L},\left(V_{v}\right)_{v \in V(\Gamma)}\right)$, where $\mathscr{L}$ is a line bundle on $\widetilde{X}_{0}$ of multidegree $w_{0}$, and each $V_{v}$ is an $(r+1)$-dimensional space of global sections of the induced line bundle $\mathscr{L}_{w_{v}}$ on $\tilde{X}_{0}$.

Denote by $G_{w}^{r}\left(\tilde{X}_{0}\right)$ the moduli scheme of pairs $(\mathscr{L}, V)$, where $\mathscr{L}$ has multidegree $w$ on $\tilde{X}_{0}$, and $V$ is an $(r+1)$-dimensional space of global sections of $\mathscr{L}$. We thus have that $\widetilde{P}_{w_{\bullet}}^{r}\left(X_{0}, \boldsymbol{n},\left(\mathscr{O}_{v}\right)_{v}\right)$ can naturally be constructed as the product over $v \in V(\Gamma)$ of the spaces $G_{w_{v}}^{r}\left(\widetilde{X}_{0}\right)$, fibered over $\operatorname{Pic}^{w_{0}}\left(\tilde{X}_{0}\right)$ via twisting by $\mathscr{O}_{w_{v}, w_{0}}$. In particular, $\widetilde{P}_{w \bullet}^{r}\left(X_{0}, \boldsymbol{n},\left(\mathscr{O}_{v}\right)_{v}\right)$ is proper over $\operatorname{Pic}^{w_{0}}\left(\widetilde{X}_{0}\right)$. 
Definition 3.7. In the situation of Notation 3.6, let $\widetilde{\mathscr{M}}$ be the universal line bundle on $\widetilde{P}_{w_{\bullet}}^{r}\left(X_{0}, \boldsymbol{n},\left(\mathscr{O}_{v}\right)_{v}\right) \times X_{0}$, and for each $w \in V\left(G\left(w_{0}\right)\right)$, let $\widetilde{\mathscr{M}}_{w}$ be induced by twisting as before. Then for each $v \in V(\Gamma)$, let $\widetilde{\mathscr{V}}_{v}$ be the universal subbundles of $p_{1 *} \widetilde{\mathscr{M}}_{w_{v}}$, and let $\widetilde{G}_{\bar{w}_{0}}^{r}\left(X / B, \boldsymbol{n},\left(\mathscr{O}_{v}\right)_{v}\right)$ be the closed subscheme of $\widetilde{P}_{w}^{r}\left(X / B, \boldsymbol{n},\left(\mathscr{O}_{v}\right)_{v}\right)$ defined by the intersection over $w \in V\left(G\left(w_{0}\right)\right)$ of the $(r+1)$ st vanishing loci of the maps

$$
p_{1 *} \widetilde{\mathscr{M}}_{w} \rightarrow \bigoplus_{v \in V(\Gamma)}\left(p_{1 *} \widetilde{\mathscr{M}}_{w_{v}}\right) / \widetilde{\mathscr{V}}_{v}
$$

Thus, a $T$-valued point of $\widetilde{P}_{w_{\bullet}}^{r}\left(X_{0}, \boldsymbol{n},\left(\mathscr{O}_{v}\right)_{v}\right)$ is a tuple $\left(\mathscr{L},\left(V_{v}\right)\right)$, where $\mathscr{L}$ is a line bundle on $T \times \widetilde{X}_{0}$ of multidegree $w_{0}$, and each $V_{v}$ is a rank- $(r+1)$ subbundle of $p_{1 *} \mathscr{L}_{w_{v}}$. Such a tuple is a $T$-valued point of $\widetilde{G}_{\bar{w}_{0}}^{r}\left(X_{0}, \boldsymbol{n},\left(\mathscr{O}_{v}\right)_{v}\right)$ if for all $w \in$ $V\left(G\left(w_{0}\right)\right)$, the map

$$
p_{1 *} \mathscr{L}_{w} \rightarrow \bigoplus_{v \in V(\Gamma)}\left(p_{1 *} \mathscr{L}_{w_{v}}\right) / V_{v}
$$

has $(r+1)$ st vanishing locus equal to all of $T$.

We now check that our two constructions are equivalent. Note that it follows in particular that $\widetilde{G}_{\bar{w}_{0}}^{r}\left(X_{0}, \boldsymbol{n},\left(\mathscr{O}_{v}\right)_{v}\right)$ is also independent of the choice of $\left(w_{v}\right)_{v}$.

Proposition 3.8. In the situation of Definition 2.21, restriction to the components $Z_{v}$ induces an isomorphism

$$
\widetilde{G}_{\bar{w}_{0}}^{r}\left(X_{0}, \boldsymbol{n},\left(\mathscr{O}_{v}\right)_{v}\right) \stackrel{\sim}{\rightarrow} G_{\bar{w}_{0}}^{r}\left(X_{0}, \boldsymbol{n},\left(\mathscr{O}_{v}\right)_{v}\right) .
$$

Proof. We first verify that restriction to the $Z_{v}$ induces a morphism

$$
\widetilde{P}_{w_{\bullet}}^{r}\left(X_{0}, \boldsymbol{n},\left(\mathscr{O}_{v}\right)_{v}\right) \rightarrow P_{w_{\bullet}}^{r}\left(X_{0}, \boldsymbol{n},\left(\mathscr{O}_{v}\right)_{v}\right),
$$

which amounts to the assertion that if $V_{v}$ is a subbundle of $p_{1 *} \mathscr{L}_{w_{v}}$ on some scheme $T$ over Spec $k$, then restricting $V_{v}$ to $Z_{v}$ induces a subbundle of the same rank of $p_{1 *} \mathscr{L}^{v}$. By Lemma B.2.3 (iii) of [Oss14c], this follows from injectivity of restriction on points, which is Proposition 3.3. Next, that (3.6) induces a morphism

$$
\widetilde{G}_{\bar{w}_{0}}^{r}\left(X_{0}, \boldsymbol{n},\left(\mathscr{O}_{v}\right)_{v}\right) \rightarrow G_{\bar{w}_{0}}^{r}\left(X_{0}, \boldsymbol{n},\left(\mathscr{O}_{v}\right)_{v}\right)
$$

is immediate from the fact that (3.2) factors through (3.5), using Corollary B.3.5 of [Oss14c].

It thus remains to prove that this morphism is an isomorphism, or equivalently that every $T$-valued point of $G_{\bar{w}_{0}}^{r}\left(X_{0}, \boldsymbol{n},\left(\mathscr{O}_{v}\right)_{v}\right)$ lifts to a unique point of $\widetilde{G}_{\bar{w}_{0}}^{r}\left(X_{0}, \boldsymbol{n},\left(\mathscr{O}_{v}\right)_{v}\right)$. Accordingly, suppose that $\left(\mathscr{L},\left(V^{v}\right)_{v \in V(\Gamma)}\right)$ is a $T$-valued point of $G_{\bar{w}_{0}}^{r}\left(X_{0}, \boldsymbol{n},\left(\mathscr{O}_{v}\right)_{v}\right)$; by the injectivity of the maps $p_{1 *} \mathscr{L}_{w_{v}} \rightarrow p_{1 * \mathscr{L}^{v}}$, a lift $\left(\mathscr{L},\left(V_{v}\right)_{v \in V(\Gamma)}\right)$ is unique, if it exists. Next, for any $v \in V(\Gamma)$, if we consider the multidegree $w_{v}$, Corollary 3.4 implies that the kernel of (3.2) is a subbundle of $p_{1 *} \mathscr{L}_{w_{v}}$ of rank $r+1$, which is the preimage of $V^{v}$. We thus set this kernel as our $V_{v}$. Thus, it is enough to see that with this choice of the bundles $V_{v}$, we have that for every multidegree $w$, the $(r+1)$ st vanishing locus of $(3.5)$ is all of $T$. But by construction, for each $v$ the natural map $\left(p_{1 *} \mathscr{L}_{w_{v}}\right) / V_{v} \rightarrow\left(p_{1 *} \mathscr{L}^{v}\right) / V^{v}$ is injective, even after arbitrary base change, so it follows that for any $w$, the kernels of (3.2) and (3.5) are identified, likewise after arbitrary base change. Then the $(r+1)$ st vanishing loci agree by Proposition B.3.4 of [Oss14c], giving the desired statement. 
We conclude this section by explaining how the construction of Definition 3.7 works in families, and applying it to prove a specialization statement.

First, the families of curves we will consider are as follows:

Definition 3.9. We say that $\pi: X \rightarrow B$ is a smoothing family if $B=\operatorname{Spec} R$ for $R$ a DVR, and further:

(I) $\pi$ is flat and proper;

(II) the special fiber $X_{0}$ of $\pi$ is a (split) nodal curve;

(III) the generic fiber $X_{\eta}$ of $\pi$ is smooth;

(IV) $\pi$ admits sections through every component of $X_{0}$.

If further $X$ is regular, we say that $\pi$ is a regular smoothing family.

See Remark 3.17 below for discussion of our choice of level of generality. Condition (IV) is always satisfied after etale base change, and is used to ensure the existence of a Picard scheme with universal line bundle.

Associated to a smoothing family we still have a dual graph $\Gamma$ : namely, the dual graph of the special fiber $X_{0}$. We then continue to use the notation $Z_{v}$ to denote the component of $X_{0}$ corresponding to a vertex $v$ of $\Gamma$. In this situation, one may define an enriched structure as before, with the additional condition that there should exist sections $s_{v}$ as in Situation 2.17. We then see:

Proposition 3.10. If $\pi: X \rightarrow B$ is a regular smoothing family, then an enriched structure is uniquely determined by setting $\mathscr{O}_{v}=\mathscr{O}_{X}\left(Z_{v}\right)$, and $s_{v}$ as in Situation 2.17 are then induced by the canonical inclusions $\mathscr{O}_{X} \rightarrow \mathscr{O}_{X}\left(Z_{v}\right)$. Moreover, this choice induces an enriched structure together with suitable sections on $X_{0}$ via restriction.

Now, we introduce the following terminology to take chain structures into account.

Definition 3.11. Given $\left(X_{0}, \boldsymbol{n}\right)$ and a regular smoothing family $\tilde{\pi}: \widetilde{X} \rightarrow \widetilde{B}$ with $\widetilde{B}$ the spectrum of a DVR, we say that $\widetilde{\pi}$ is of fiber type $\left(X_{0}, \boldsymbol{n}\right)$ if the special fiber of $\tilde{\pi}$ is isomorphic to (a base extension of) the curve $\widetilde{X}_{0}$ obtained from $\left(X_{0}, \boldsymbol{n}\right)$. Given also a smoothing family $\pi: X \rightarrow B$, with special fiber $X_{0}$, we say that $\tilde{\pi}$ is an extension of $\pi$ if it is obtained from $\pi$ via base extension followed by iterated blowups at the nodes of the special fiber.

Whenever we say $\pi$ is of fiber type $\left(X_{0}, \boldsymbol{n}\right)$, we implicitly assume that we have fixed an isomorphism between the special fiber of $\pi$ and the appropriate base extension of $\tilde{X}_{0}$.

(Regular) smoothing families of type $\left(X_{0}, \boldsymbol{n}\right)$ arise naturally in two different ways: the first is as extensions of a given regular smoothing family $\pi$, taken for instance in order to extend the generic point to a field of definition of a line bundle on the geometric generic fiber, in which case the line bundle will extend over the extended family. The second is as regularizations of irregular families, in which case no base change is involved. The former will be more immediately important to us, but our theory is general enough to handle both situations at once.

Situation 3.12. Suppose $\tilde{\pi}: \widetilde{X} \rightarrow \widetilde{B}$ is a regular smoothing family of fiber type $\left(X_{0}, \boldsymbol{n}\right)$, and we also fix an admissible multidegree $w_{0}$ on $\widetilde{X}_{0}$, as well as a tuple $\left(w_{v}\right)_{v \in V(\widetilde{\Gamma})}$ of vertices of $G\left(w_{0}\right)$, with each $w_{v}$ concentrated at $v$. Let $\left(\mathscr{O}_{v}, s_{v}\right)_{v \in V(\Gamma)}$ be the enriched structure and associated sections on $\tilde{X}_{0}$ given by Proposition 3.10. 
In Situation 3.12, given $w \in V\left(G\left(w_{0}\right)\right)$, denote by $\operatorname{Pic}^{w}(\widetilde{X} / \widetilde{B})$ the moduli schemes of line bundles of degree $d$ which have multidegree $w$ on fibers lying over the closed point of $\widetilde{B}$. Then denote by $G_{w}^{r}(\widetilde{X} / \widetilde{B})$ the moduli scheme of pairs $(\mathscr{L}, V)$, where $\mathscr{L}$ is in $\operatorname{Pic}^{w}(\widetilde{X} / \widetilde{B})$, and $V$ is an $(r+1)$-dimensional space of global sections of $\mathscr{L}$. The representability of these spaces is standard; one can argue just as in the proof of Theorem 5.3 of [Oss06], for instance. The maps (3.4) generalize to this situation, and we can then generalize the previous constructions to the case of families.

Notation 3.13. Construct $\widetilde{P}_{w \bullet}^{r}\left(\widetilde{X} / \widetilde{B}, X_{0}, \boldsymbol{n},\left(\mathscr{O}_{v}\right)_{v}\right)$ as the product over $v \in V(\Gamma)$ of the spaces $G_{w_{v}}^{r}(\tilde{X})$, fibered over $\operatorname{Pic}^{w_{0}}(\tilde{X})$, and let $\widetilde{G}_{\bar{w}_{0}}^{r}\left(\tilde{X} / \widetilde{B}, X_{0}, \boldsymbol{n},\left(\mathscr{O}_{v}\right)_{v}\right)$ be the closed subscheme defined by the intersection of the $(r+1)$ st vanishing loci of the maps (3.4), as $w$ varies over $V\left(G\left(w_{0}\right)\right)$.

We then have the following basic fact.

Proposition 3.14. The moduli scheme $\widetilde{G}_{\bar{w}_{0}}^{r}\left(\tilde{X} / \widetilde{B}, X_{0}, \boldsymbol{n},\left(\mathscr{O}_{v}\right)_{v}\right)$ is proper over $\operatorname{Pic}^{w_{0}}(\tilde{X} / \widetilde{B})$. Its generic fiber is naturally identified with $G_{d}^{r}\left(X_{\eta}\right)$, and its special fiber with (the appropriate base extension of) $\widetilde{G}_{\bar{w}_{0}}^{r}\left(X_{0}, \boldsymbol{n},\left(\mathscr{O}_{v}\right)_{v}\right)$.

Proof. The first statement is immediate from the construction, as is the statement on the special fiber. The description of the generic fiber follows from the observation that the maps $f_{w, w^{\prime}}$ are all isomorphisms over the generic fiber; in fact, we claim that if we fix any $v$, then an arbitrary choice of $V_{v}$ uniquely determines $V_{v^{\prime}}$ as the image of $V_{v}$ for all $v^{\prime} \neq v$. Indeed, using Proposition B.3.4 and Lemma B.2.3 (iv) of [Oss14c], we see that for a given choice of $V_{v}$, if we consider $w=w_{v}$ we will have the desired condition on the $(r+1)$ st vanishing locus of $(3.5)$ if and only if $V_{v}$ maps into each of the $V_{v^{\prime}}$, which is the same as saying that $V_{v^{\prime}}$ is the image of $V_{v}$. On the other hand, if $V_{v^{\prime}}$ is the image of $V_{v}$ for all $v^{\prime}$, we see that the kernel of (3.5) for any $w$ is simply the image of $V_{v}$, so we have the desired behavior of the $(r+1)$ st vanishing locus.

Corollary 3.15. Let $\pi: X \rightarrow B$ be a smoothing family, with special fiber $X_{0}$. Let $(\mathscr{L}, V)$ be a $\mathfrak{g}_{d}^{r}$ on the geometric generic fiber $X_{\bar{\eta}}$. Then there exists a chain structure $\boldsymbol{n}$ on $X_{0}$, an extension $\tilde{\pi}: \widetilde{X} \rightarrow \widetilde{B}$ of $\pi$ having fiber type $\left(X_{0}, \boldsymbol{n}\right)$, and an admissible multidegree $w_{0}$ on the resulting $\tilde{X}_{0}$ such that $\mathscr{L}$ extends to a line bundle of multidegree $w_{0}$ on $\tilde{X}$.

For any such $\boldsymbol{n}, \tilde{\pi}$, and $w_{0}$, and any collection of $w_{v} \in V\left(G\left(w_{0}\right)\right)$ concentrated at each $v \in V(\Gamma)$, we have that $(\mathscr{L}, V)$ extends to a limit linear series on $\tilde{X}$.

Proof. This is mostly standard, but also brief, so we include it for the convenience of the reader. The last assertion is immediate from Propositions 3.14 and 3.8.

For the first assertion, we necessarily have $(\mathscr{L}, V)$ defined over some finite extension $\eta^{\prime}$ of $\eta$; let $B^{\prime}$ be the corresponding integral closure of $B$, localized at a closed point. If $X^{\prime}=X \times_{B} B^{\prime}$, then if we repeatedly blow up the non-smooth locus of $X^{\prime}$ over $B^{\prime}$ to obtain a regular total space, we obtain our $\boldsymbol{n}$ and $\tilde{X}$. Since $\mathscr{L}$ is now defined over the new generic fiber, and $\tilde{X}$ is still regular, we can extend $\mathscr{L}$ to all of $\tilde{X}$. It remains to see that the extension can be chosen to have admissible multidegree, but this is easily achieved by twisting first at non-exceptional components to achieve sufficiently positive degree on each chain of exceptional components, and then twisting at exceptional components first to achieve nonnegativity on each component, and then admissibility. 
Remark 3.16. In fact, we see from the proof of Corollary 3.15 that we have the following refined statement: let $\boldsymbol{n}^{\prime}$ be the chain structure on $X_{0}$ obtained by setting $\boldsymbol{n}^{\prime}(e)$ to be one greater than the number of blowups required to make make $X$ regular at the point corresponding to $e$. Then the $\boldsymbol{n}$ produced in the proof is of the form $\boldsymbol{n}(e)=m \boldsymbol{n}^{\prime}(e)$ for all $e$, where $m$ is the ramification index of $B^{\prime}$ over $B$.

Thus, the collection of chain structures we need to consider in order to extend line bundles on the initial family are not arbitrary, but are restricted to multiples of the "base" chain structure $\boldsymbol{n}^{\prime}$.

Remark 3.17. The base $B$ in Definition 3.9 may be generalized considerably, but this makes the conditions more complicated; compare Definitions 2.1.1 and 2.2.2 of [Oss14c]. Moreover, imposing the existence of an enriched structure will imply that even if $B$ is higher-dimensional, the geometry of the family all occurs in codimension 1 , so there seems to be little reason to introduce additional technical complications.

\section{The TWO-COMPOnEnT CASE}

In order to give the equivalent definition which will ultimately generalize that of Eisenbud and Harris, the two-component case is the simplest situation to consider. Conveniently, it is also the base case of an induction argument for the more general situation, so we will first develop the key comparison result for curves with two components. In this case, we simplify our notation as follows.

Situation 4.1. Let $X_{0}$ consist of two smooth curves $Z_{1}, Z_{2}$ glued to one another at nodes $P_{1}, \ldots, P_{m}$. Fix the orientation on $\Gamma$ with all edges going from $Z_{1}$ to $Z_{2}$. Let $\boldsymbol{n}$ be a chain structure, and for $i=1, \ldots, m$, write $n_{i}:=\boldsymbol{n}\left(P_{i}\right)$. For $i=1, \ldots, m$, and $j=1, \ldots, n_{i}-1$, let $E_{i, j}$ denote the $j$ th exceptional component of $\widetilde{X}_{0}$ lying over $P_{i}$ on $X_{0}$. Fix an admissible multidegree $w_{0}$ on $\left(X_{0}, \boldsymbol{n}\right)$, and multidegrees $w_{1}, w_{2} \in V\left(G\left(w_{0}\right)\right)$ concentrated at $Z_{1}, Z_{2}$ respectively. Write $\mu_{i}:=\mu_{1}\left(P_{i}\right)$, where $w_{1}=\left(\left(w_{1}\right)_{\Gamma}, \mu_{1}(\bullet)\right)$. Let $b$ be the number of twists at $Z_{1}$ required to get from $w_{1}$ to $w_{2}$. Identify $V\left(G\left(w_{0}\right)\right)$ with $\mathbb{Z}$ by sending $w$ to the number of twists at $Z_{1}$ required to get from $w_{1}$ to $w$.

We will assume throughout this section that we are in the above situation. In this case, $G\left(w_{0}\right)$ is an unbounded chain, with edges going in each direction. We have identified $w_{1}$ with 0 , and $w_{2}$ with $b$. Accordingly, for any line bundle $\mathscr{L}$ of multidegree $w_{0}$ on $\widetilde{X}_{0}$, for $i \in \mathbb{Z}$ we will write $\mathscr{L}_{i}$ for the line bundle $\mathscr{L}_{w}$, where $w$ is obtained from $w_{1}$ by twisting $i$ times at $Z_{1}$. As we have already done above, when convenient we will write nodes or components in place of the corresponding edges or vertices of the dual graph.

We then introduce the following notation as well.

Notation 4.2. For any line bundle $\mathscr{L}$ of multidegree $w_{0}$ on $\tilde{X}_{0}$, write $\mathscr{L}^{1}:=\left.\mathscr{L}_{0}\right|_{Z_{1}}$, and $\mathscr{L}^{2}:=\left.\mathscr{L}_{b}\right|_{Z_{2}}$.

We now define sequences of effective divisors supported on the $P_{i}$ which will be used to give multivanishing sequences.

Definition 4.3. Let $D_{0}^{1}, \ldots, D_{b+1}^{1}$ be the sequence of effective divisors on $Z_{1}$ defined by $D_{0}^{1}=0$, and for $i \geqslant 0$,

$$
D_{i+1}^{1}-D_{i}^{1}=\sum_{j: \mu_{j}+i \equiv 0} P_{\left(\bmod n_{j}\right)},
$$


and similarly define $D_{0}^{2}, \ldots, D_{b+1}^{2}$ on $Z_{2}$ by $D_{0}^{2}=0$, and for $i \geqslant 0$,

$$
D_{i+1}^{2}-D_{i}^{2}=\sum_{j: \mu_{j}+b-i \equiv 0} P_{\left(\bmod n_{j}\right)} .
$$

The relationship between the twisting divisors and line bundles is given by the following basic proposition, whose proof is left to the reader.

Proposition 4.4. For $i=0, \ldots, b+1$, we have

$$
\left.\mathscr{L}_{i}\right|_{Z_{1}}=\mathscr{L}^{1}\left(-D_{i}^{1}\right) \quad \text { and }\left.\mathscr{L}_{i-1}\right|_{Z_{2}}=\mathscr{L}^{2}\left(-D_{b+1-i}^{2}\right),
$$

where we use equality to denote canonical isomorphism.

We also have for all $i=0, \ldots, b$ that $P_{j}$ is in the support of $D_{i+1}^{1}-D_{i}^{1}$ if and only if $\mathscr{L}_{i}$ has degree 0 on $E_{j, \ell}$ for all $\ell$, and $P_{j}$ is in the support of $D_{i+1}^{2}-D_{i}^{2}$ if and only if $\mathscr{L}_{b-i}$ has degree 0 on $E_{j, \ell}$ for all $\ell$. In particular, if $E_{i}$ denotes the union over $j$ such that $P_{j}$ in the support of $D_{i+1}^{1}-D_{i}^{1}$ of the chains of exceptional components lying over the $P_{j},\left.\mathscr{L}_{i}\right|_{E_{i}} \cong \mathscr{O}_{E_{i}}$, and we thus get an induced isomorphism

$$
\varphi_{i}: \mathscr{L}^{1}\left(-D_{i}^{1}\right) / \mathscr{L}^{1}\left(-D_{i+1}^{1}\right) \stackrel{\sim}{\rightarrow} \mathscr{L}^{2}\left(-D_{b-i}^{2}\right) / \mathscr{L}^{2}\left(-D_{b+1-i}^{2}\right)
$$

for each $i$.

We think of the $\varphi_{i}$ as being gluing maps; in the case of trivial chain structure, the $\varphi_{i}$ are each defined on all nodes at once, but in general they are only defined on subsets of the nodes, which depend on $i$.

Definition 4.5. In the situation of Definition 1.2, we say that $j$ is critical for $D$. if $D_{j+1} \neq D_{j}$.

Our main comparison result in the two-component case is as then follows:

Lemma 4.6. In Situation 4.1, fix also an enriched structure on $\tilde{X}_{0}$, and sections $s_{v}$ as in Situation 2.17. For a given $\left(\mathscr{L},\left(V^{1}, V^{2}\right)\right)$, and $i=1,2$, denote by $a_{0}^{i}, \ldots, a_{r}^{i}$ the multivanishing sequence of $V^{i}$ along the $D_{\bullet}^{i}$. Then $\left(\mathscr{L},\left(V^{1}, V^{2}\right)\right)$ is a limit linear series if and only if

(I) for $\ell=0, \ldots, r$, if $a_{\ell}^{1}=\operatorname{deg} D_{j}^{1}$ with $j$ critical for $D_{\bullet}^{1}$, then

$$
a_{r-\ell}^{2} \geqslant \operatorname{deg} D_{b-j}^{2} \text {; }
$$

(II) for $i=1,2$, there exist bases $s_{0}^{i}, \ldots, s_{r}^{i}$ of the $V^{i}$ such that

$$
\operatorname{ord}_{D .} s_{\ell}^{i}=a_{\ell}^{i} \quad \text { for } \ell=0, \ldots, r
$$

and for all $\ell$ with (4.1) an equality, we have

$$
\varphi_{j}\left(s_{\ell}^{1}\right)=s_{r-\ell}^{2}
$$

when we consider $s_{\ell}^{1} \in V^{1}\left(-D_{j}^{1}\right)$ and $s_{r-\ell}^{2} \in V^{2}\left(-D_{b-j}^{2}\right)$, with $j$ as in (I).

Remark 4.7. Although condition (I) appears asymmetric, in fact this is not the case; indeed, Proposition 4.4 says that the construction of the $D_{\bullet}^{i}$ implies that $j$ is critical for $D_{\bullet}^{1}$ if and only if $b-j$ is critical for $D_{\bullet}^{2}$, so (I) is equivalent to requiring that if $a_{r-\ell}^{2}=\operatorname{deg} D_{b-j}^{2}$ with $b-j$ critical for $D_{\bullet}^{2}$, then $a_{\ell}^{1} \geqslant \operatorname{deg} D_{j}^{1}$.

As an intermediate step, it is convenient to consider a bounded version of $G\left(w_{0}\right)$ as follows. 
Notation 4.8. Let $\bar{G}\left(w_{0}\right)$ denote the directed subgraph of $G\left(w_{0}\right)$ consisting of all vertices between $w_{1}$ and $w_{2}$ (inclusive), and with all edges of $G\left(w_{0}\right)$ connecting vertices in $V\left(\bar{G}\left(w_{0}\right)\right)$.

It turns out that in the definition of limit linear series, considering multidegrees in $\bar{G}\left(w_{0}\right)$ suffices.

Proposition 4.9. In the situation of Lemma 4.6, $\left(\mathscr{L},\left(V^{1}, V^{2}\right)\right)$ is a limit linear series if and only if (2.1) has kernel of dimension at least $r+1$ for all $w \in V\left(\bar{G}\left(w_{0}\right)\right)$.

Proof. Since $V\left(\bar{G}\left(w_{0}\right)\right) \subseteq V\left(G\left(w_{0}\right)\right)$, one direction is trivial. Conversely, suppose that (2.1) has kernel of dimension at least $r+1$ for all $w \in V\left(\bar{G}\left(w_{0}\right)\right)$, and let $w^{\prime} \in V\left(G\left(w_{0}\right)\right)$ be arbitrary; we need to show that (2.1) also has kernel of dimension at least $r+1$ in multidegree $w^{\prime}$. Considering $w^{\prime}=i$ for some $i \in \mathbb{Z}$, there are three cases to consider: either $0 \leqslant i \leqslant b$, or $i<0$, or $i>b$. The first case is the same as having $w^{\prime} \in V\left(\bar{G}\left(w_{0}\right)\right)$, so there is nothing to show. The other two cases being symmetric, we only treat the case that $i<0$. In this case, we claim that the kernel $W$ of (2.1) in multidegree $w_{1}$ injects into the kernel of (2.1) in multidegree $w^{\prime}$ under $f_{w_{1}, w^{\prime}}$. Indeed, it is clear that the entire image of $f_{w_{1}, w^{\prime}}$ is contained in the kernel of (2.1), so it suffices to see that $f_{w_{1}, w^{\prime}}$ is injective on $W$. But $f_{w_{1}, w^{\prime}}$ is induced by a map which is an inclusion on $Z_{1}$, so the desired injectivity is an immediate consequence of Proposition 3.3.

Next, in $\bar{G}\left(w_{0}\right)$, we can reinterpret the kernel of (2.1) as follows.

Proposition 4.10. In the situation of Lemma 4.6, for $i=0, \ldots, b$, consider the map

$$
V^{1}\left(-D_{i}^{1}\right) \oplus V^{2}\left(-D_{b-i}^{2}\right) \rightarrow \mathscr{L}^{2}\left(-D_{b-i}^{2}\right) / \mathscr{L}^{2}\left(-D_{b-i+1}^{2}\right)
$$

induced by taking quotients, and applying $-\varphi_{i}$ on the first factor. Then our morphisms $H^{0}\left(\tilde{X}_{0}, \mathscr{L}_{i}\right) \rightarrow H^{0}\left(Z_{j}, \mathscr{L}^{j}\right)$ for $j=1,2$ induce an isomorphism between the kernel of (2.1) and the kernel of (4.2).

Proof. The image of $H^{0}\left(\tilde{X}_{0}, \mathscr{L}_{i}\right)$ in $H^{0}\left(Z_{1}, \mathscr{L}^{1}\right)$ (respectively, $\left.H^{0}\left(Z_{2}, \mathscr{L}^{2}\right)\right)$ is contained in $H^{0}\left(Z_{1}, \mathscr{L}^{1}\left(-D_{i}^{1}\right)\right)$ (respectively, $H^{0}\left(Z_{2}, \mathscr{L}^{2}\left(-D_{b-i}^{2}\right)\right)$ ) by construction, so a section of $H^{0}\left(X_{0}, \mathscr{L}_{i}\right)$ which lies in the kernel of (2.1) necessarily restricts to $V^{1}\left(-D_{i}^{1}\right)$ on $Z_{1}$ and $V^{2}\left(-D_{b-i}^{2}\right)$ on $Z_{2}$. That it in fact yields an element in the kernel of (4.2) is essentially the definition of $\varphi_{i}$. To see that the constructed map is bijective, the main point is that given a pair $\left(s_{1}, s_{2}\right) \in H^{0}\left(Z_{1},\left.\mathscr{L}_{i}\right|_{Z_{1}}\right) \oplus H^{0}\left(Z_{2},\left.\mathscr{L}_{i}\right|_{Z_{2}}\right)$, an extension of $\left(s_{1}, s_{2}\right)$ to a global section $s \in H^{0}\left(\tilde{X}_{0}, \mathscr{L}_{i}\right)$ is unique if it exists, and it exists if and only if $\varphi_{i}\left(s_{1}\right)=s_{2}$, using the identifications of Proposition 4.4. Indeed, the assertion is clear on the union of exceptional chains $E_{i}$ from the construction of $\varphi_{i}$, so it is enough to check that there is always a unique extension over the exceptional chains not contained in the $E_{i}$. But if $E$ is such a chain, then $\left.\mathscr{L}_{i}\right|_{E}$ has degree 1 on exactly one irreducible component, and degree 0 on the others, and it follows that $\left.\mathscr{L}_{i}\right|_{E}$ has a unique global section with arbitrary prescribed values at either end of $E$, giving the desired assertion. The desired bijectivity follows.

We can now finish our examination of the two-component case.

Proof of Lemma 4.6. First, by Propositions 4.9 and 4.10, we have reduced to showing that $\left(\mathscr{L},\left(V^{1}, V^{2}\right)\right)$ satisfies (I) and (II) if and only if (4.2) has kernel of dimension at least $r+1$ for $i=0, \ldots, b$. 
Now, observe that

$\operatorname{dim} V^{1}\left(-D_{i}^{1}\right)=\#\left\{\ell: a_{\ell}^{1} \geqslant \operatorname{deg} D_{i}^{1}\right\}$, and $\operatorname{dim} V^{2}\left(-D_{b-i}^{2}\right)=\#\left\{\ell: a_{\ell}^{2} \geqslant \operatorname{deg} D_{b-i}^{2}\right\}$.

For each $i$, let $r_{i}$ be the rank of (4.2); note that $r_{i}=0$ unless $i$ is critical for $D_{\text {. }}^{1}$. Choose $\ell_{1}$ and $\ell_{3}$ minimal with $a_{\ell_{1}}^{1} \geqslant \operatorname{deg} D_{i}^{1}$ and $a_{\ell_{3}}^{2} \geqslant \operatorname{deg} D_{b-i}^{2}$, and $\ell_{2}$ and $\ell_{4}$ maximal with with $a_{\ell_{2}}^{1} \leqslant \operatorname{deg} D_{i}^{1}$ and $a_{\ell_{4}}^{2} \leqslant \operatorname{deg} D_{b-i}^{2}$. Here, if $a_{\ell}^{1}<\operatorname{deg} D_{i}^{1}$ for all $\ell$, set $\ell_{1}=r+1$, if $a_{\ell}^{1}>\operatorname{deg} D_{i}^{1}$ for all $\ell$, set $\ell_{2}=-1$, and similarly for $\ell_{3}$ and $\ell_{4}$. Then the kernel of (4.2) has dimension equal to

$$
\operatorname{dim} V^{1}\left(-D_{i}^{1}\right)+\operatorname{dim} V^{2}\left(-D_{b-i}^{2}\right)-r_{i}=r+1-\ell_{1}+r+1-\ell_{3}-r_{i},
$$

so for the kernel of (4.2) to have dimension at least $r+1$ is equivalent to

$$
\ell_{1}+\ell_{3}+r_{i} \leqslant r+1 \text {. }
$$

In addition, we see that condition (II) of the lemma is equivalent to having that, for each critical $i$ for $D_{\bullet}^{1}$, the images of $V^{1}\left(-D_{i}^{1}\right)$ and $V^{2}\left(-D_{b-i}^{2}\right)$ under (4.2) overlap with dimension at least equal to

$$
\#\left\{\ell: \ell_{1} \leqslant \ell \leqslant \ell_{2} \text {, and } \ell_{3} \leqslant r-\ell \leqslant \ell_{4}\right\} .
$$

Now, if we assume condition (I), we claim that for all $i$, we have $\ell_{1}+\ell_{3} \leqslant r+1$, and that if $i$ is critical for $D_{\bullet}^{1}$, we also have $\ell_{3} \leqslant r-\ell_{2}$ and $\ell_{1} \leqslant r-\ell_{4}$. For the first claim, note that by definition $a_{\ell_{1}-1}^{1}<\operatorname{deg} D_{i}^{1}$; if we let $i^{\prime}$ be critical for $D_{\bullet}^{1}$ with $a_{\ell_{1}-1}^{1}=\operatorname{deg} D_{i^{\prime}}^{1}$, then (I) implies that $a_{r+1-\ell_{1}}^{2} \geqslant \operatorname{deg} D_{b-i^{\prime}}^{2} \geqslant \operatorname{deg} D_{b-i}^{2}$, so $\ell_{3} \leqslant r+1-\ell_{1}$, giving the first claimed inequality. Next, if $i$ is critical for $D_{\bullet}^{1}$, then there are two cases to consider: if $\operatorname{deg} D_{i}^{1}$ does not occur in $a^{1}$, we will have $\ell_{2}=\ell_{1}-1$, and in this case the inequality $\ell_{3} \leqslant r-\ell_{2}$ is the same as $\ell_{3} \leqslant r+1-\ell_{1}$, which we have just proved. On the other hand, if $\operatorname{deg} D_{i}^{1}$ does occur in $a^{1}$, then (I) gives $a_{r-\ell_{2}}^{2} \geqslant D_{b-i}^{2}$, which means $\ell_{3} \leqslant r-\ell_{2}$, as desired. The proof of the last claimed inequality $\ell_{1} \leqslant r-\ell_{4}$ is similar, taking into account Remark 4.7.

Still assuming (I), we next claim that (4.2) having kernel of dimension at least $r+1$ for $i=0, \ldots, b$ is equivalent to condition (II). If $i$ is not critical for $D_{\bullet}^{1}$, then $r_{i}=0$, so we see from (4.3) that the desired kernel size follows from the inequality $\ell_{1}+\ell_{3} \leqslant r+1$, which we have already proved. If $i$ is critical for $D_{\bullet}^{1}$, using $\ell_{3} \leqslant r-\ell_{2}$ and $\ell_{1} \leqslant r-\ell_{4}$. the inequalities in (4.4) simplify to $r-\ell_{4} \leqslant \ell \leqslant \ell_{2}$. Thus, the existence of the desired basis is equivalent to requiring that the images of $V^{1}\left(-D_{i}^{1}\right)$ and $V^{2}\left(-D_{b-i}^{2}\right)$ under (4.2) overlap with dimension at least equal to $\ell_{2}+1-\left(r-\ell_{4}\right)$. On the other hand, the dimension of this overlap is equal to the sum of the dimensions of the images of $V^{1}\left(-D_{i}^{1}\right)$ and $V^{2}\left(-D_{b-i}^{2}\right)$, minus $r_{i}$, which is to say, $\ell_{2}+1-\ell_{1}+\ell_{4}+1-\ell_{3}-r_{i}$, so we conclude that (again, assuming (I)), condition (II) is equivalent to the inequality

$$
\ell_{2}+1-\ell_{1}+\ell_{4}+1-\ell_{3}-r_{i} \geqslant \ell_{2}+1-\left(r-\ell_{4}\right),
$$

which is the same as (4.3). This proves the claim, and we conclude that (I) and (II) together imply that $\left(\mathscr{L},\left(V^{1}, V^{2}\right)\right)$ is a linear linear series, and moreover, that to see the converse, it is enough to prove that (4.3) implies condition (I).

Thus, assume (4.3). Given $\ell \in\{0, \ldots, r\}$, let $i$ be critical for $D_{\bullet}^{1}$ with $\operatorname{deg} D_{i}^{1}=a_{\ell}^{1}$, and choose $\ell_{1}, \ell_{2}, \ell_{3}, \ell_{4}$ as above. Observe that $r_{i} \geqslant \#\left\{\ell^{\prime}: a_{\ell^{\prime}}^{1}=i\right\}=\ell_{2}+1-\ell_{1}$ so that (I) implies that $r+1-\ell_{1}-\ell_{3} \geqslant \ell_{2}+1-\ell_{1}$. It thus follows that $r \geqslant \ell_{2}+\ell_{3} \geqslant \ell+\ell_{3}$, so $r-\ell \geqslant \ell_{3}$. Thus, we find

$$
a_{r-\ell}^{2} \geqslant a_{\ell_{3}}^{2} \geqslant \operatorname{deg} D_{b-i}^{2},
$$


giving (I), and completing the proof of the lemma.

\section{The PSEUdoCOMPACT-TYPE CASE}

We conclude by generalizing the results of the previous section to arbitrary curves of pseudocompact type, thereby providing a simultaneous generalization of the twocomponent case and the compact-type case. As before, we start with combinatorial preliminaries.

Notation 5.1. If $\Gamma$ is a graph, let $\bar{\Gamma}$ be the graph obtained from $\Gamma$ by collapsing all multiple edges to single edges, while leaving the vertex set unchanged. We say $\Gamma$ is a multitree if $\bar{\Gamma}$ is a tree.

Just as before we defined twists motivated by twisting at a component, in the multitree case we define twists motivated by twisting on one side or the other of the node(s) at which two components meet.

Definition 5.2. If $\Gamma$ is a multitree, and $(e, v)$ a pair of an edge $e$ and an adjacent vertex $v$ of $\bar{\Gamma}$, given an admissible multidegree $w$, we define the twist of $w$ at $(e, v)$ to be obtained from $w$ as follows: for each $\tilde{e}$ of $\Gamma$ over $e$, increase $\mu(\tilde{e})$ by $\sigma(\tilde{e}, v)$. Now, decrease $w_{\Gamma}(v)$ by the number of $\tilde{e}$ for which $\mu(\tilde{e})$ had been equal to 0 , and for each $\tilde{e}$, if the new $\mu(\tilde{e})$ is zero, increase $w_{\Gamma}\left(v^{\prime}\right)$ by 1 , where $v^{\prime}$ is the other vertex adjacent to $v$.

Notice that if $v^{\prime}$ is the other vertex adjacent to an edge $e$, then twisting at $\left(e, v^{\prime}\right)$ is inverse to twisting at $(e, v)$. In addition, we observe that the twist of $w$ at $(e, v)$ may be obtained as a sequence of twists of $w$ at vertices $v^{\prime}$, where $v^{\prime}$ varies over the set of vertices in the same connected component as $v$ in $\bar{\Gamma} \backslash\{e\}$. Conversely, twisting of $w$ at any $v$ can also be obtained as a composition of twists at $(e, v)$, where $e$ varies over edges adjacent to $v$.

Throughout this section, all twists will be with respect to pairs $(e, v)$, rather than vertices.

Warning 5.3. Even though on a combinatorial level, twisting $w$ by $v$ can be obtained by a sequence of twists at different $(e, v)$, the same does not hold on the level of the maps between the associated line bundles.

Situation 5.4. Suppose we are given a multitree $\Gamma$, and an admissible multidegree $w_{0}$, and let $\left(w_{v}\right)_{v \in V(\Gamma)}$ be a collection of elements of $V\left(G\left(w_{0}\right)\right)$ such that:

(I) each $w_{v}$ is concentrated at $v$;

(II) for each $v, v^{\prime} \in V(\bar{\Gamma})$ connected by an edge $e$, the multidegree $w_{v^{\prime}}$ is obtained from $w_{v}$ by twisting $b_{v, v^{\prime}}$ times at $(e, v)$, for some $b_{v, v^{\prime}} \in \mathbb{Z}_{\geqslant 0}$.

Definition 5.5. In Situation 5.4, let $V\left(\bar{G}\left(w_{0}\right)\right) \subseteq V\left(G\left(w_{0}\right)\right)$ consist of admissible multidegrees $w$ such that there exist $v, v^{\prime} \in V(\bar{\Gamma})$ connected by some edge $e$, with $w$ obtainable from $w_{v}$ by twisting $b$ times at $(e, v)$, for some $b$ with $0 \leqslant b \leqslant b_{v, v^{\prime}}$.

There is an edge $\epsilon$ from from $w$ to $w^{\prime}$ in $\bar{G}\left(w_{0}\right)$ if there exist $(e, v)$ in $\bar{\Gamma}$ such that $w^{\prime}$ is obtained from $w$ by twisting at $(e, v)$.

Thus, $\bar{G}\left(w_{0}\right)$ is a tree, obtained by subdividing every edge of $\bar{\Gamma}$ into $b_{v, v^{\prime}}$ edges, and replacing each edge with a pair of directed edges in opposite directions. Note that in general the edges of $\bar{G}\left(w_{0}\right)$ need not be edges of $G\left(w_{0}\right)$, but can be thought of as "compositions" of edges of $G\left(w_{0}\right)$. However, in the case that $\Gamma$ has only two 
vertices, we have that $G\left(w_{0}\right)$ and $\bar{G}\left(w_{0}\right)$ are both chains, with the only difference being that $\bar{G}\left(w_{0}\right)$ is bounded by $w_{v_{1}}$ and $w_{v_{2}}$, while $G\left(w_{0}\right)$ is unbounded. Thus, our notation is consistent with that of Notation 4.8.

We now move on to the geometric definitions and statements.

Definition 5.6. Let $X_{0}$ be a projective nodal curve, with dual graph $\Gamma$. $X_{0}$ is of pseudocompact type if $\Gamma$ is a multitree.

Situation 5.7. In Situation 5.4, suppose also that our $\Gamma$ is obtained as the dual graph of a given projective nodal curve $X_{0}$.

Notation 5.8. In Situation 5.7, for each pair $(e, v)$ of an edge and adjacent vertex of $\bar{\Gamma}$, let $D_{0}^{(e, v)}, \ldots, D_{b_{v, v^{\prime}}+1}^{(e, v)}$ be the sequence of effective divisors on $Z_{v}$ defined by $D_{0}^{(e, v)}=0$, and for $i \geqslant 0$,

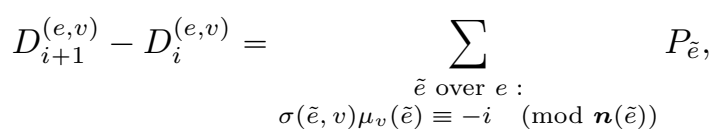

where $P_{\tilde{e}}$ denotes the node of $X_{0}$ corresponding to $\tilde{e}$, and $\mu_{v}(\bullet)$ is obtained from $w_{v}$.

Our main result is the following.

Theorem 5.9. In the situation of Definition 2.21, suppose further that $X_{0}$ is of pseudocompact type, and we are in Situation 5.7. Then given a tuple $\left(\mathscr{L},\left(V^{v}\right)_{v \in V(\Gamma)}\right)$, for each pair $(e, v)$ in $\bar{\Gamma}$, let $a_{0}^{(e, v)}, \ldots, a_{r}^{(e, v)}$ be the multivanishing sequence of $V^{v}$ along $D_{\bullet}^{(e, v)}$. Then the following are equivalent:

(a) $\left(\mathscr{L},\left(V^{v}\right)_{v}\right)$ is a limit linear series;

(b) (2.1) has kernel of dimension at least $r+1$ for every $w \in V\left(\bar{G}\left(w_{0}\right)\right)$;

(c) for any $e \in E(\Gamma)$, with adjacent vertices $v, v^{\prime}$, we have:

(I) for $\ell=0, \ldots, r$, if $a_{\ell}^{(e, v)}=\operatorname{deg} D_{j}^{(e, v)}$ with $j$ critical for $D_{\bullet}^{(e, v)}$, then

$$
a_{r-\ell}^{\left(e, v^{\prime}\right)} \geqslant \operatorname{deg} D_{b_{v, v^{\prime}}-j}^{\left(e, v^{\prime}\right)}
$$

(II) there exist bases $s_{0}^{(e, v)}, \ldots, s_{r}^{(e, v)}$ of $V^{v}$ and $s_{0}^{\left(e, v^{\prime}\right)}, \ldots, s_{r}^{\left(e, v^{\prime}\right)}$ of $V^{v^{\prime}}$ such that

$$
\operatorname{ord}_{D_{\bullet}^{(e, v)}} s_{\ell}^{(e, v)}=a_{\ell}^{(e, v)}, \quad \text { for } \ell=0, \ldots, r,
$$

and similarly for $s_{\ell}^{\left(e, v^{\prime}\right)}$, and for all $\ell$ with (5.1) an equality, we have

$$
\varphi_{j}^{(e, v)}\left(s_{\ell}^{(e, v)}\right)=s_{r-\ell}^{\left(e, v^{\prime}\right)}
$$

when we consider $s_{\ell}^{(e, v)} \in V^{v}\left(-D_{j}^{(e, v)}\right)$ and $s_{r-\ell}^{\left(e, v^{\prime}\right)} \in V^{v^{\prime}}\left(-D_{b_{v, v^{\prime}}-j}^{\left(e, v^{\prime}\right)}\right)$, where $j$ is as in (I), and $\varphi_{j}$ is as in Proposition 4.4.

In (II) above, note that although Proposition 4.4 was only stated for twocomponent curves, since we are only interested in a given pair of adjacent vertices of $\Gamma$, the situation is no different in our present more general case.

We first introduce some convenient notation. The following can be used to keep track of twisting at nodes: 
Notation 5.10. In Situation 5.4, given $w \in V\left(G\left(w_{0}\right)\right)$, and $(e, v)$ adjacent in $\bar{\Gamma}$, let $t_{(e, v)}(w)$ be the number of twists at $(e, v)$ required to go from $w_{v}$ to $w$ in a minimal number of twists.

Note that $t_{(e, v)}(w)$ is well-defined, since the only way to cancel a twist at $(e, v)$ is to twist at $\left(e, v^{\prime}\right)$, where $v^{\prime}$ is the other vertex adjacent to $v$. In addition, we have $t_{(e, v)}(w)+t_{\left(e, v^{\prime}\right)}(w)=b_{v, v^{\prime}}$.

We can now define a notion of restriction of multidegrees to subcurves. Of course, one can always restrict naively, but this turns out not to be well behaved with respect to limit linear series, so instead we make the following definition.

Definition 5.11. In Situation 5.4, let $X_{0}^{\prime}$ be a connected subcurve of $X_{0}$, with dual graph $\Gamma^{\prime}$. Then for any $w \in V\left(G\left(w_{0}\right)\right)$, define the restriction of $w$ to $X_{0}^{\prime}$ as follows: starting from $w$, let $w^{\prime}$ be the admissible multidegree obtained by, for each pair $(e, v)$ in $\bar{\Gamma}$ where $v \in \Gamma^{\prime}$ but the other vertex $v^{\prime}$ adjacent to $e$ is not in $\Gamma^{\prime}$, twisting $t_{(e, v)}(w)$ times at $\left(e, v^{\prime}\right)$. Then, the restriction of $w$ to $X_{0}^{\prime}$ is the naive restriction of $w^{\prime}$.

The reason for this choice of restriction, rather than the more naive one, is that if we naively restrict an arbitrary $w$, it will no longer be obtainable as a twist of the restrictions of the $w_{v}$. With our choice of restriction, even though we modify $w$, we will be able to understand the kernel of (2.1) for a given $w$ in terms of the kernels of the restrictions to subcurves covering $X_{0}$; see the proof of Theorem 5.9 below.

Note that if $w \in V\left(\bar{G}\left(w_{0}\right)\right)$, say between $w_{v}$ and $w_{v^{\prime}}$, and if $X_{0}^{\prime}$ contains $Z_{v}$ and $Z_{v^{\prime}}$, then in fact the restriction of Definition 5.11 is simply the same as naive restriction.

Proof of Theorem 5.9. First observe that because $V\left(\bar{G}\left(w_{0}\right)\right) \subseteq V\left(G\left(w_{0}\right)\right)$, the implication (a) implies (b) is trivial. We will prove that (b) implies (c) and (c) implies (a), by induction on the number of components of $X_{0}$. The base case is that $X_{0}$ has two components, which is precisely Lemma 4.6, together with Proposition 4.9.

Now, for the induction step, the basic observation is that condition (c) is imposed on a pair of nodes at a time, so that (c) holds if and only if for each pair $v_{1}, v_{2}$ of adjacent vertices of $\Gamma$, the restriction $\left(\mathscr{L}_{w_{0}^{\prime}} \mid Z_{v_{1}} \cup Z_{v_{2}},\left(V^{v_{1}}, V^{v_{2}}\right)\right)$ also satisfies (c) for the curve $Z_{v_{1}} \cup Z_{v_{2}}$, where $w_{0}^{\prime}$ is any element of $V\left(\bar{G}\left(w_{0}\right)\right)$ lying between $w_{v_{1}}$ and $w_{v_{2}}$. Note that $\left.\operatorname{deg} \mathscr{L}_{w_{0}^{\prime}}\right|_{z_{v_{1}}} \cup Z_{v_{2}}$ is not in general equal to $d$, but is independent of the choice of $w_{0}^{\prime}$.

Thus, to see that (b) implies (c), we suppose that (2.1) has kernel of dimension at least $r+1$ for every $w \in V\left(\bar{G}\left(w_{0}\right)\right)$, and we will show that if $v_{1}, v_{2} \in V(\Gamma)$ are adjacent, then $\left(\mathscr{L}_{w_{0}^{\prime}} \mid Z_{v_{1}} \cup Z_{v_{2}},\left(V^{v_{1}}, V^{v_{2}}\right)\right)$ satisfies (c). But suppose $w \in V\left(\bar{G}\left(w_{0}\right)\right)$ lies between $w_{v_{1}}$ and $w_{v_{2}}$. Then note that $w$ agrees with both $w_{v_{1}}$ and $w_{v_{2}}$ away from $v_{1}$ and $v_{2}$ and the edges between them, so arguing as in Proposition 3.3, the kernel of (2.1) for $X_{0}$ injects into the kernel of (2.1) for $Z_{v_{1}} \cup Z_{v_{2}}$ under restriction to $Z_{v_{1}} \cup Z_{v_{2}}$. Thus, by Lemma 4.6 we conclude that $\left(\mathscr{L}_{w_{0}^{\prime}} Z_{v_{1}} \cup Z_{v_{2}},\left(V^{v_{1}}, V^{v_{2}}\right)\right)$ satisfies (c), as desired.

On the other hand, if (c) is satisfied, we prove the desired statement by induction on the number of components. Given $w \in V\left(G\left(w_{0}\right)\right)$, there are two cases to consider. First, if for some $(e, v)$, we have $t_{(e, v)}(w)<0$, let $X_{0}^{\prime}$ be the subcurve of $X_{0}$ corresponding to the connected component $\bar{\Gamma} \backslash\{e\}$ containing $v$. Then if $w^{\prime}$ is the 
multidegree obtained from $w$ in Definition 5.11, because $t_{(e, v)}(w)<0$, we have a map $\mathscr{L}_{w^{\prime}} \rightarrow \mathscr{L}_{w}$ which is injective on $\tilde{X}_{0}^{\prime}$; let $Y$ be the subcurve of $\tilde{X}_{0}$ on which it is injective, and $Z$ the subcurve on which it vanishes. Thus, $\tilde{X}_{0}^{\prime} \subseteq Y$, and $\tilde{X}_{0}=Y \cup Z$, and also $Y$ and $Z$ have no components in common. We thus have an inclusion $\left.\left.\mathscr{L}_{w^{\prime}}\right|_{Y} \rightarrow \mathscr{L}_{w}\right|_{Y}$ whose image vanishes at $Y \cap Z$, and it follows that we can extend by zero to get an inclusion

$$
H^{0}\left(Y,\left.\mathscr{L}_{w^{\prime}}\right|_{Y}\right) \hookrightarrow H^{0}\left(\tilde{X}_{0}, \mathscr{L}_{w}\right)
$$

On the other hand, by construction we observe that $\mathscr{L}_{w^{\prime}}$ is trivial on components on $Y$ not contained in $\tilde{X}_{0}^{\prime}$, so we have

$$
H^{0}\left(\widetilde{X}_{0}^{\prime},\left.\mathscr{L}_{w^{\prime}}\right|_{\widetilde{X}_{0}^{\prime}}\right)=H^{0}\left(Y,\left.\mathscr{L}_{w^{\prime}}\right|_{Y}\right)
$$

inducing an inclusion

$$
H^{0}\left(\widetilde{X}_{0}^{\prime},\left.\mathscr{L}_{w^{\prime}}\right|_{\widetilde{X}_{0}^{\prime}}\right) \hookrightarrow H^{0}\left(\tilde{X}_{0}, \mathscr{L}_{w}\right)
$$

Now, we have by hypothesis that (c) is satisfied on $\tilde{X}_{0}^{\prime}$, so by the induction hypothesis, the kernel of (2.1) for $\tilde{X}_{0}^{\prime}$ has dimension at least $r+1$ in multidegree $w^{\prime}$, and using the above inclusion, we get the same for $\tilde{X}_{0}$ in multidegree $w$, as desired.

The second case is that $t_{(e, v)}(w) \geqslant 0$ for all $(e, v)$, in which case we necessarily have $0 \leqslant t_{(e, v)}(w) \leqslant b_{v, v^{\prime}}$. In this case, choose $v_{1} \in V(\Gamma)$ which is only adjacent to one other $v_{2} \in V(\Gamma)$ (i.e., which is a leaf of $\bar{\Gamma}$ ). Let $X_{0}^{\prime}$ be the closure of the complement of $Z_{v_{1}}$ in $X_{0}$; then by hypothesis, (c) is satisfied for the restrictions $\left(\left.\mathscr{L}_{w_{0}^{\prime}}\right|_{z_{v_{1}} \cup Z_{v_{2}}},\left(V^{v}, V^{v_{2}}\right)\right)$ and $\left(\left.\mathscr{L}_{w_{0}^{\prime \prime}}\right|_{X_{0}^{\prime}},\left(V^{v}\right)_{v \neq v_{1}}\right)$, where $w_{0}^{\prime \prime}$ is any element of $V\left(\bar{G}\left(w_{0}\right)\right)$ not lying between $w_{v_{1}}$ and $w_{v_{2}}$. By the induction hypothesis, we conclude that (2.1) has kernel of dimension at least $r+1$ for $Z_{v_{1}} \cup Z_{v_{2}}$ in multidegree $w^{\prime}$ and for $X_{0}^{\prime}$ in multidegree $w^{\prime \prime}$, where $w^{\prime}$ and $w^{\prime \prime}$ are the restrictions of $w$. But because $0 \leqslant t_{(e, v)}(w) \leqslant b_{v, v^{\prime}}$ for all $(e, v)$, the kernel of (2.1) for $X_{0}$ in multidegree $w$ is simply the fibered product of the above two kernels over $V^{v_{2}}$, and hence also has dimension at least $r+1$, as desired.

\section{A smoothing TheOREM}

In this section, we prove the following theorem, which says that - just as in the Eisenbud-Harris case - when the space of limit linear series on a curve of pseudocompact type has the expected dimension, then every limit linear series arises as the limit of linear series on smooth curves. In fact, our theorem is stronger even in the compact-type case, as it is not restricted to refined limit linear series. Our proof is fundamentally different from that of Eisenbud and Harris, although it still relies in the end on obtaining a lower bound on the dimension of a relative moduli space. The key ingredient is the theory of linked determinantal loci, developed in Appendix A. We also use a portion of Theorem 5.9, in essence to reduce to the two-component case.

Theorem 6.1. Let $\pi: X \rightarrow B$ be a smoothing family, with special fiber $X_{0} a$ curve of pseudocompact type. Let $\boldsymbol{n}$ be a chain structure on $X_{0}$, and $\widetilde{\pi}: \widetilde{X} \rightarrow \widetilde{B}$ an extension of $\pi$ having fiber type $\left(X_{0}, \boldsymbol{n}\right)$. Let $\left(\mathscr{O}_{v}\right)_{v}$ be the induced enriched structure on $X_{0}$.

Given an admissible multidegree $w_{0}$ on the resulting $\tilde{X}_{0}$, and $\left(w_{v}\right)_{v}$ as in Situation 5.4, if the moduli space $G_{\bar{w}_{0}}^{r}\left(X_{0}, \boldsymbol{n},\left(\mathscr{O}_{v}\right)_{v}\right)$ has dimension $\rho$ at a given point, 
then the corresponding limit linear series arises as the limit of linear series on the geometric generic fiber of $\pi$.

More precisely, if $\pi: \widetilde{X} \rightarrow \widetilde{B}$ is any regular smoothing family of fiber type $\left(X_{0}, \boldsymbol{n}\right)$, then the scheme $\widetilde{G}_{\bar{w}_{0}}^{r}\left(\widetilde{X} / \widetilde{B}, X_{0}, \boldsymbol{n},\left(\mathscr{O}_{v}\right)_{v}\right)$ has universal relative dimension at least $\rho$ over $B$, and if the special fiber $G_{\bar{w}_{0}}^{r}\left(X_{0}, \boldsymbol{n},\left(\mathscr{O}_{v}\right)_{v}\right)$ has dimension exactly $\rho$ at a point, then $\widetilde{G}_{\bar{w}_{0}}^{r}\left(\tilde{X} / \widetilde{B}, X_{0}, \boldsymbol{n},\left(\mathscr{O}_{v}\right)_{v}\right)$ is universally open at that point. If also the special fiber is geometrically reduced at the given point, then $\widetilde{G}_{\bar{w}_{0}}^{r}\left(\widetilde{X} / \widetilde{B}, X_{0}, \boldsymbol{n},\left(\mathscr{O}_{v}\right)_{v}\right)$ is flat at that point.

In the above, we use the relative dimension terminology introduced in [Oss13].

Proof. The idea is to give a slightly different construction of the relative limit linear series moduli space $\widetilde{G}_{\bar{w}_{0}}^{r}\left(\widetilde{X} / \widetilde{B}, X_{0}, \boldsymbol{n},\left(\mathscr{O}_{v}\right)_{v}\right)$, taking ideas from the proof of Theorem 5.3 of [Oss06] and using the linked determinantal loci developed in Appendix A. We can work set-theoretically, since our goal is a dimension statement. As in our earlier construction, start with the scheme $\operatorname{Pic}^{w_{0}}\left(\widetilde{X}_{0}\right)$, which is smooth over $B$ of relative dimension $g$, and let $\widetilde{\mathscr{M}}$ be the universal line bundle, with $\widetilde{\mathscr{M}}_{w}$ the induced line bundle in multidegree $w$ for each $w \in V\left(G\left(w_{0}\right)\right)$. Next, choose a sufficiently $\pi$-ample divisor $D$ on $\widetilde{X}$; using our sections of $\pi$, we may assume that $D=\sum_{v \in V(\Gamma)} D_{v}$, where $D_{v} \cap X_{0}$ meets only $Z_{v}$. Note that we do not need to twist up on the exceptional components, since they are rational and our multidegrees are always nonnegative on them. We then have for each $w$ that $p_{1 *}\left(\widetilde{\mathscr{M}}_{w}(D)\right)$ is locally free of rank $d+\operatorname{deg} D+1-g$, and commutes with base change. Let $G$ be the fibered product over $\operatorname{Pic}^{w_{0}}\left(\tilde{X}_{0}\right)$ of the schemes $G\left(r+1, p_{1 *}\left(\widetilde{\mathscr{M}}_{w_{v}}(D)\right)\right)$, where $v$ ranges over $V(\Gamma)$. This is thus smooth over $B$ of relative dimension

$$
\begin{aligned}
g+|V(\Gamma)|(r+1)(d+\operatorname{deg} D+1-g- & (r+1)) \\
& =g+|V(\Gamma)|(r+1)(d+\operatorname{deg} D-r-g) .
\end{aligned}
$$

For each $v$, let $\mathscr{V}_{v}$ be (the pullback to $G$ of) the universal subbundle of $p_{1 *}\left(\widetilde{\mathscr{M}}_{w_{v}}(D)\right)$. Let $G^{\prime}$ be the closed subset of $G$ obtained by imposing that for each $v$, the composed map

$$
\mathscr{V}_{v} \rightarrow p_{1 *}\left(\widetilde{\mathscr{M}}_{w_{v}}(D)\right) \rightarrow p_{1 *}\left(\left.\widetilde{\mathscr{M}}_{w_{v}}(D)\right|_{D_{v}}\right)
$$

vanishes identically, and by intersecting, for each $e \in E(\bar{\Gamma})$ having adjacent vertices $v, v^{\prime}$, with the linked determinantal locus associated to the chain $p_{1 *}\left(\widetilde{\mathscr{M}}_{w}(D)\right)$ for $w$ between $w_{v}$ and $w_{v^{\prime}}$ together with the subbundles $\mathscr{V}_{v}$ and $\mathscr{V}_{v^{\prime}}$. Then our key claim is that $G^{\prime}$ is equal to $\widetilde{G}_{\bar{w}_{0}}^{r}\left(\tilde{X} / \widetilde{B}, X_{0}, \boldsymbol{n},\left(\mathscr{O}_{v}\right)_{v}\right)$. Given the claim, we are done: the former conditions impose codimension at most $(r+1)\left(\sum_{v} \operatorname{deg} D_{v}\right)=(r+1) \operatorname{deg} D$, and the latter impose, by Theorem A.3, codimension at most

$$
\begin{aligned}
|E(\bar{\Gamma})|(r+1)(d+\operatorname{deg} D+1-g-(r & +1)) \\
& =(|V(\Gamma)|-1)(r+1)(d+\operatorname{deg} D-r-g) .
\end{aligned}
$$

Subtracting the above maximal codimensions from the relative dimension of $G$, we are left with $g+(r+1)(d-r-g)=\rho$, and according to Corollary 5.1 of [Oss13], we find that $\widetilde{G}_{\bar{w}_{0}}^{r}\left(\tilde{X} / \widetilde{B}, X_{0}, \boldsymbol{n},\left(\mathscr{O}_{v}\right)_{v}\right)$ has universal relative dimension at least $\rho$ over $B$, as desired. The assertions on universal openness and flatness in the case that the special fiber has dimension exactly $\rho$ at a point then follow from Proposition 3.7 of [Oss13]. 
We are thus reduced to proving the claim. On the level of points, we analyze first the generic fiber $X_{\eta}$, and then the special fiber $\tilde{X}_{0}$. Over the generic fiber, the maps between the $\mathscr{L}_{w}$ are all isomorphisms, so the linked determinantal conditions in the definition of $G^{\prime}$ imply that the $V_{v}$ all map to one another under these isomorphisms, and the condition that each $V_{v}$ vanish on $D_{v}$ implies that they all vanish on all of $D$. Thus, for a fixed choice of $v$, we have that points of $G^{\prime}$ on the generic fiber are all uniquely determined by a choice of $V_{v}$ contained in $\mathscr{L}_{w_{v}}$, which is the same as $\widetilde{G}_{\bar{w}_{0}}^{r}\left(\tilde{X} / \widetilde{B}, X_{0}, \boldsymbol{n},\left(\mathscr{O}_{v}\right)_{v}\right)$. Next, on the special fiber, we are asserting the following: given a line bundle $\mathscr{L}$ of multidegree $w_{0}$ and a tuple $\left(V_{v}\right)_{v}$ with $V_{v} \subseteq \Gamma\left(\tilde{X}_{0}, \mathscr{L}_{w_{v}}(D)\right)$, if each $V_{v}$ vanishes on $D_{v}$, and for each $w \in V\left(\bar{G}\left(w_{0}\right)\right)$ between $w_{v}$ and $w_{v^{\prime}}$, the map

$$
\Gamma\left(\widetilde{X}_{0}, \mathscr{L}_{w}(D)\right) \rightarrow \Gamma\left(\widetilde{X}_{0}, \mathscr{L}_{w_{v}}(D)\right) / V_{v} \oplus \Gamma\left(\tilde{X}_{0}, \mathscr{L}_{w_{v^{\prime}}}(D)\right) / V_{v^{\prime}}
$$

has kernel of dimension at least $r+1$, then in fact each $V_{v}$ is contained in $\Gamma\left(\tilde{X}_{0}, \mathscr{L}_{w_{v}}\right)$, and the map

$$
\Gamma\left(\tilde{X}_{0}, \mathscr{L}_{w}\right) \rightarrow \bigoplus_{v} \Gamma\left(\tilde{X}_{0}, \mathscr{L}_{w_{v}}\right) / V_{v}
$$

has kernel of dimension at least $r+1$ for all $w \in V\left(G\left(w_{0}\right)\right)$. Our first observation is that for all $v, v^{\prime}$, we must have $V_{v}$ mapping into $V_{v^{\prime}} \subseteq \Gamma\left(\widetilde{X}_{0}, \mathscr{L}_{w_{v^{\prime}}}(D)\right)$ under the natural twisting maps. Because the maps $\mathscr{L}_{w_{v}} \rightarrow \mathscr{L}_{w_{v^{\prime}}}$ always factor as a sequence of such maps between adjacent vertices, it is enough to prove this when $v, v^{\prime}$ are adjacent. In this case, we consider (6.1) in the case $w=w_{v}$, noting that the kernel is necessarily contained in $V_{v}$. Then our hypothesis implies that the kernel is all of $V_{v}$, and hence that $V_{v}$ maps into $V_{v^{\prime}}$, as desired. Our next observation is that for $w \in V\left(\bar{G}\left(w_{0}\right)\right)$, under our hypotheses we have that the kernel of (6.1) is identified with the kernel of

$$
\Gamma\left(\tilde{X}_{0}, \mathscr{L}_{w}(D)\right) \rightarrow \bigoplus_{v^{\prime \prime}} \Gamma\left(\tilde{X}_{0}, \mathscr{L}_{w_{v^{\prime \prime}}}(D)\right) / V_{v^{\prime \prime}}
$$

Indeed, this follows from the first observation, together with the fact that if $w$ lies between $w_{v}$ and $w_{v^{\prime}}$, then for any $v^{\prime \prime}$ the map $\mathscr{L}_{w} \rightarrow \mathscr{L}_{w_{v^{\prime \prime}}}$ always factors through either $\mathscr{L}_{w_{v}}$ or $\mathscr{L}_{w_{v}}$.

It then follows that the kernel of (6.3) vanishes on $D$ for each $w$, since for each $v$, the map $\mathscr{L}_{w} \rightarrow \mathscr{L}_{w_{v}}$ is injective on $Z_{v}$, so if $V_{v}$ vanishes on $D_{v}$ the kernel of (6.3) vanishes on $D_{v}$ as well. Since the $D_{v}$ are disjoint, we conclude that the kernel vanishes on $D$. Considering the case $w=w_{v}$, we conclude in particular that each $V_{v}$ vanishes on $D$, as desired. It follows that the kernel of (6.3) is identified with the kernel of (6.2), so we have proved the desired statement for $w \in V\left(\bar{G}\left(w_{0}\right)\right)$. Moreover, if we set $V^{v}$ to be the image of $V_{v}$ in $\Gamma\left(Z_{v}, \mathscr{L}^{v}\right)$, we see that the kernel of (6.2) is identified with the kernel of (2.1), so the equivalence of (a) and (b) in Theorem 5.9 then yields the desired statement for all $w \in V\left(G\left(w_{0}\right)\right)$.

Remark 6.2. Note that despite the pseudocompact type hypothesis, our proof of the smoothing theorem was built around our general definition of limit linear series rather than the equivalent definition of $\S 5$. In fact, we expect that a similar proof should be possible in full generality, with the main difficulty being the need for a much more general theory of linked Grassmannians. In our proof, due to the special form of curves of pseudocompact type, we were able to inductively reduce to what was, in essense, the "two-component" version of the linked Grassmannian, 
but in general no such reduction is possible. There is some evidence, in the form of examples and of parallel results for local models of certain Shimura varieties (see, for instance, Goertz [Goe01]), that such a general theory of linked Grassmannians should exist, but we expect that it will be substantially more difficult than the special case we have used here.

We conclude with a scheme structure comparison result involving the construction carried out in the proof of Theorem 6.1. This relates our construction to the related definitions for the higher-rank case given in $\S 4.2$ of [Oss14c], and more importantly will be used in [MO] to prove a comparison theorem in the rank-1, compact type case between our scheme structure and the scheme structure given by the Eisenbud-Harris definition.

Notation 6.3. Now suppose that we are in the situation of Theorem 6.1 , or of Theorem 5.9 , in which case we take $B=\widetilde{B}$ to be a point. Let $\widetilde{G}_{\bar{w}_{0}}^{\prime r}\left(\widetilde{X} / \widetilde{B}, X_{0}, \boldsymbol{n},\left(\mathscr{O}_{v}\right)_{v}\right)$ be the closed subscheme of the space $\widetilde{P}_{w \cdot}^{r}\left(\widetilde{X} / \widetilde{B}, X_{0}, \boldsymbol{n},\left(\mathscr{O}_{v}\right)_{v}\right)$ defined by the intersection of the $(r+1)$ st vanishing loci of the maps $(3.4)$, as $w$ varies over $V\left(\bar{G}\left(w_{0}\right)\right)$.

Thus, a priori we have that $\widetilde{G}_{\bar{w}_{0}}^{r}\left(\tilde{X} / \widetilde{B}, X_{0}, \boldsymbol{n},\left(\mathscr{O}_{v}\right)_{v}\right)$ is a closed subscheme of $\widetilde{G}_{\bar{w}_{0}}^{\prime r}\left(\tilde{X} / \widetilde{B}, X_{0}, \boldsymbol{n},\left(\mathscr{O}_{v}\right)_{v}\right)$, and Theorem 5.9 tells us that they are supported on the same subset.

Proposition 6.4. The moduli scheme $\widetilde{G}_{\bar{w}_{0}}^{\prime r}\left(\widetilde{X} / \widetilde{B}, X_{0}, \boldsymbol{n},\left(\mathscr{O}_{v}\right)_{v}\right)$ is proper over $\operatorname{Pic}^{w_{0}}(\tilde{X} / \widetilde{B})$, and in the case that $\pi$ is a smoothing family, its generic fiber is naturally identified with $G_{d}^{r}\left(X_{\eta}\right)$.

Moreover, the set-theoretic construction of $\widetilde{G}_{\bar{w}_{0}}^{r}\left(\widetilde{X} / \widetilde{B}, X_{0}, \boldsymbol{n},\left(\mathscr{O}_{v}\right)_{v}\right)$ described in the proof of Theorem 6.1 yields a scheme structure agreeing with $\widetilde{G}_{\bar{w}_{0}}^{\prime r}\left(\widetilde{X} / \widetilde{B}, X_{0}, \boldsymbol{n},\left(\mathscr{O}_{v}\right)_{v}\right)$.

Proof. The proof of the first part is the same as for Proposition 3.14. For the second part, we need to show that the set-theoretic analysis in the proof of Theorem 6.1 works on the level of $T$-valued points if we consider only $w \in V\left(\bar{G}\left(w_{0}\right)\right)$. Thus, suppose we are given a $T$-valued tuple $\left(\mathscr{L},\left(V_{v}\right)_{v}\right)$, where each $V_{v}$ is a subbundle of $p_{1 *} \mathscr{L}_{w_{v}}(D)$, and for any $w \in V\left(\bar{G}\left(w_{0}\right)\right)$ between $w_{v}$ and $w_{v^{\prime}}$, the map

$$
p_{1 *} \mathscr{L}_{w}(D) \rightarrow\left(\left(p_{1 *} \mathscr{L}_{w_{v}}(D)\right) / V_{v}\right) \oplus\left(\left(p_{1 *} \mathscr{L}_{w_{v^{\prime}}}(D)\right) / V_{v^{\prime}}\right)
$$

has $(r+1)$ st vanishing locus equal to $T$, and also that the composed maps

$$
V_{v} \rightarrow p_{1 *} \mathscr{L}_{w_{v}}(D) \rightarrow p_{1 *}\left(\left.\mathscr{L}_{w_{v}}(D)\right|_{D_{v}}\right.
$$

are zero for each $v$. We want to show that in fact all the $V_{v}$ vanish on all of $D$, and for all $w \in V\left(\bar{G}\left(w_{0}\right)\right)$, the $(r+1)$ st vanishing locus of $(3.5)$ is all of $T$.

First, given $v, v^{\prime}$ adjacent, setting $w=w_{v}$ in (6.4), we see by Proposition B.3.4 and Lemma B.2.3 (iv) of [Oss14c] that the kernel must be equal to $V_{v}$, and thus that $V_{v}$ maps into $V_{v^{\prime}}$. Traversing $\bar{\Gamma}$ in this way we conclude that each $V_{v}$ maps into each $V_{v^{\prime}}$ for any $v^{\prime} \neq v$. We then observe that for any $w$, and any $v^{\prime}$, the map $p_{1 *}\left(\left.\mathscr{L}_{w}(D)\right|_{D_{v^{\prime}}}\right) \rightarrow p_{1 *}\left(\left.\mathscr{L}_{w_{v^{\prime}}}(D)\right|_{D_{v^{\prime}}}\right)$ is an isomorphism, so since $V_{v}$ maps into $V_{v^{\prime}}$ and $V_{v^{\prime}}$ vanishes on $D_{v^{\prime}}$, we conclude that $V_{v}$ likewise vanishes on $D_{v^{\prime}}$. Since $D=\sum_{v^{\prime}} D_{v^{\prime}}$, we find that each $V_{v}$ vanishes on all of $D$, and may be considered as a subbundle of $p_{1 *} \mathscr{L}_{w_{v}}$. Similarly, we see that the kernel of (6.4) is (universally) identified with the kernel of

$$
p_{1 *} \mathscr{L}_{w} \rightarrow\left(\left(p_{1 *} \mathscr{L}_{w_{v}}\right) / V_{v}\right) \oplus\left(\left(p_{1 *} \mathscr{L}_{w_{v^{\prime}}}\right) / V_{v^{\prime}}\right)
$$


so by Proposition B.3.2 of [Oss14c] we have that the $(r+1)$ st vanishing loci of the two maps agree. But then, again using that each $V_{v}$ maps into each other $V_{v^{\prime}}$, and the map from $\mathscr{L}_{w}$ to $\mathscr{L}_{w_{v^{\prime \prime}}}$ factors through $\mathscr{L}_{w_{v}}$ or $\mathscr{L}_{w_{v^{\prime}}}$ if $w$ lies between $w_{v}$ and $w_{v^{\prime}}$, we see that the kernel of (6.5) is also universally identified with the kernel of (3.5), giving the desired statement.

Note that neither the construction from Theorem 6.1 nor our analysis of its scheme structure depended on $B$ being positive-dimensional, and in particular we also conclude the desired statement in the case that $B$ is a point.

\section{Appendix A. Linked Determinantal LOCI}

In this appendix, we develop a theory of "linked determinantal loci," which are in essence a determinantal locus analogue of the linked Grassmannian developed in Appendix A of [Oss06]. A preliminary definition is the following:

Definition A.1. Let $S$ be a scheme, and $d, n$ be positive integers. Suppose that $\mathscr{E}_{1}, \ldots, \mathscr{E}_{n}$ are vector bundles of rank $d$ on $S$ and we have morphisms

$$
f_{i}: \mathscr{E}_{i} \rightarrow \mathscr{E}_{i+1}, \quad f^{i}: \mathscr{E}_{i+1} \rightarrow \mathscr{E}_{i}
$$

for each $i=1, \ldots, n-1$. Given $s \in \Gamma\left(S, \mathscr{O}_{S}\right)$, we say that $\mathscr{E}_{\bullet}=\left(\mathscr{E}_{i}, f_{i}, f^{i}\right)_{i}$ is an $s$-linked chain if the following conditions are satisfied:

(I) For each $i=1, \ldots, n$,

$$
f_{i} \circ f^{i}=s \cdot \mathrm{id} \text {, and } f^{i} \circ f_{i}=s \cdot \mathrm{id} .
$$

(II) On the fibers of the $\mathscr{E}_{i}$ at any point with $s=0$, we have that for each $i=1, \ldots, n-1$,

$$
\operatorname{ker} f^{i}=\operatorname{im} f_{i} \text {, and } \operatorname{ker} f_{i}=\operatorname{im} f^{i} .
$$

(III) On the fibers of the $\mathscr{E}_{i}$ at any point with $s=0$, we have that for each $i=1, \ldots, n-2$,

$$
\operatorname{im} f_{i} \cap \operatorname{ker} f_{i+1}=(0) \text {, and } \operatorname{im} f^{i+1} \cap \operatorname{ker} f^{i}=(0) .
$$

This is precisely the condition required for the ambient chain of vector bundles in the definition of a linked Grassmannian in [Oss06], although the terminology was introduced later, in [OT14]. We then define:

Definition A.2. Let $\mathscr{E}_{\bullet}$ be an $s$-linked chain on a scheme $S$. Given $r>0$, suppose $\mathscr{F}_{1}, \mathscr{F}_{n}$ are rank- $r$ subbundles of $\mathscr{E}_{1}$ and $\mathscr{E}_{n}$ respectively. Then the associated linked determinantal locus is the closed subscheme of $S$ on which the morphisms

$$
\mathscr{E}_{i} \rightarrow\left(\mathscr{E}_{1} / \mathscr{F}_{1}\right) \oplus\left(\mathscr{E}_{n} / \mathscr{F}_{n}\right)
$$

have rank less than or equal to $d-r$ for all $i=1, \ldots, n$.

In Definition A.2, the necessary morphisms $\mathscr{E}_{i} \rightarrow \mathscr{E}_{j}$ are obtained simply by composing the $f_{i}$ or $f^{i}$, as appropriate.

Thus, a linked determinantal locus is by definition an intersection of $n$ determinal loci in $S$, for morphisms from vector bundles of rank $d$ to vector bundles of rank $2 d-2 r$. The standard codimension bound for determinantal loci then implies that (each irreducible component of) a linked determinantal locus has codimension at most $n(d-(d-r))(2 d-2 r-(d-r))=n r(d-r)$. However, the structure imposed by our hypotheses implies that in fact, the codimension is far smaller. Our main theorem is the following. 
Theorem A.3. Each irreducible component of a linked determinantal locus has codimension at most $r(d-r)$ in $S$.

Remark A.4. Notice that set-theoretically, the linked determinantal locus is the set of points of $S$ at which the kernel of (A.1) has dimension at least $r$, or equivalently, the set of points such that the fiber of $\mathscr{E}_{i}$ contains at least an $r$-dimensional space which maps into $\mathscr{F}_{1}$ inside $\mathscr{E}_{1}$ and into $\mathscr{F}_{n}$ inside $\mathscr{E}_{n}$. In particular, the case $i=1$ implies that on the linked determinantal locus, we must have $\mathscr{F}_{1}$ mapping into $\mathscr{F}_{n}$, and the $i=n$ case implies that $\mathscr{F}_{n}$ must map into $\mathscr{F}_{1}$.

Now, in order to see that Theorem A.3 is plausible, consider points of $S$ over which $s$ is nonzero. On this locus, all the maps are isomorphisms, and our hypotheses imply that $\mathscr{F}_{1}$ maps into $\mathscr{F}_{n}$ if and only if $\mathscr{F}_{n}$ maps into $\mathscr{F}_{1}$, and that moreover the linked determinantal locus consists precisely of the points on which $\mathscr{F}_{1}$ maps into $\mathscr{F}_{n}$. Hence, on this locus it is clear that the codimension is at most $r(d-r)$, and we see that the interesting part of the theorem is the locus on which $s$ vanishes, or, crucially for our application to smoothing theorems, the global situation in which $s$ vanishes at some points but not others.

The strategy of our proof parallels the proof of the corresponding statement for determinantal varieties: we first consider the universal case and conclude the desired statement by realizing the linked determinantal locus as the image of a linked Grassmannian, and then conclude the statement of the theorem by pulling back from the universal case.

We next recall the definition of the linked Grassmannian.

Definition A.5. Let $S$ be a scheme, $\mathscr{E}_{\bullet}$ an $s$-linked chain on $S$, and $r>0$. Then the linked Grassmannian $\mathrm{LG}\left(r, \mathscr{E}_{\bullet}\right)$ is the closed subscheme of

$$
G\left(r, \mathscr{E}_{1}\right) \times_{S} \cdots \times_{S} G\left(r, \mathscr{E}_{n}\right)
$$

consisting of tuples $\left(\mathscr{F}_{1}, \ldots, \mathscr{F}_{n}\right)$ such that for $i=1, \ldots, n-1$ we have $f_{i}\left(\mathscr{F}_{i}\right) \subseteq$ $\mathscr{F}_{i+1}$ and $f^{i}\left(\mathscr{F}_{i+1}\right) \subseteq \mathscr{F}_{i}$.

The relationship between linked Grassmannians and linked determinantal loci is described by the following proposition.

Proposition A.6. Let $S_{0}$ be any scheme, and $\overline{\mathscr{E}}_{\bullet}$ an s-linked chain on $S_{0}$. Let $S=G\left(r, \overline{\mathscr{E}}_{1}\right) \times_{S_{0}} G\left(r, \overline{\mathscr{E}}_{n}\right)$, and let $\mathscr{E}_{\bullet}$ be the pullback of $\overline{\mathscr{E}}_{\bullet}$ to $S$, with $\mathscr{F}_{1} \subseteq \mathscr{E}_{1}$ and $\mathscr{F}_{n} \subseteq \mathscr{E}_{n}$ the pullbacks of the universal bundles on $G\left(r, \overline{\mathscr{E}}_{1}\right)$ and $G\left(r, \overline{\mathscr{E}}_{n}\right)$ respectively.

Then the linked determinantal locus associated to $\mathscr{E}_{\bullet}$ and $\mathscr{F}_{1}, \mathscr{F}_{n}$ is precisely the image of the linked Grassmannian $\mathrm{LG}\left(r, \overline{\mathscr{E}}_{\bullet}\right)$ under the projection morphism

$$
G\left(r, \mathscr{E}_{1}\right) \times_{S_{0}} \cdots \times_{S_{0}} G\left(r, \mathscr{E}_{n}\right) \rightarrow G\left(r, \mathscr{E}_{1}\right) \times_{S_{0}} G\left(r, \mathscr{E}_{n}\right) .
$$

Proof. It is clear from the definitions that the image of $\operatorname{LG}\left(r, \overline{\mathscr{E}}_{\bullet}\right)$ is contained in the linked determinantal locus, so we need only prove the converse. Since the statement is set-theoretic, we may work on the level of $k$-valued points with $k$ a field, and we see that what we want to prove is the following: given $d$-dimensional $k$-vector spaces $E_{1}, \ldots, E_{n}$, maps $f^{i}$ and $f_{i}$ making an $s$-linked chain on Spec $k$, and $r$-dimensional subspaces $F_{1} \subseteq E_{1}$ and $F_{n} \subseteq E_{n}$ such that the kernel of (A.1) has dimension at least $r$ for $i=1, \ldots, n$, then there exist choices of $r$-dimensional subspaces $F_{i} \subseteq E_{i}$ for $i=2, \ldots, n-1$ which are linked by the $f_{i}$ and $f^{i}$.

Now, let $K_{i} \subseteq E_{i}$ be the kernel of (A.1) for $i=2, \ldots, n-1$. Then by hypothesis, $\operatorname{dim} K_{i} \geqslant r$ for all $i$, and it is also clear that $f_{i}\left(K_{i}\right) \subseteq K_{i+1}$ and $f^{i}\left(K_{i+1}\right) \subseteq K_{i}$ for 
all $i$. We claim that as long as $\operatorname{dim} K_{i}>r$ for some $i$, we can replace some $K_{i}$ by a proper subspace while preserving the above conditions; iterating this process yields the desired statement. Now, let $i$ be minimal such that $\operatorname{dim} K_{i}>r$; we claim that the span of the images of $K_{i-1}$ and $K_{i+1}$ in $K_{i}$ must be strictly smaller than $K_{i}$. Indeed, by condition (III) of $s$-linkage, the image of $K_{i+1}$ in $K_{i}$ also injects into $K_{i-1}$, but maps into the kernel of $f_{i-1}$. Because $\operatorname{dim} K_{i-1}=r$, we conclude that the span of the images of $K_{i-1}$ and $K_{i+1}$ in $K_{i}$ must have dimension at most $r$, so we can replace $K_{i}$ by any $r$-dimensional subspace containing this span; this will preserve the linkage condition, and thus proves the claim.

We next need to set up the relevant universal spaces. We have the following:

Proposition A.7. Given $d>0$, let $\bar{U}_{d}$ be the scheme of pairs of $d \times d$ matrixes $A$ and $B$ over $\mathbb{Z}[t]$ such

$$
A B=B A=t I_{d} .
$$

Let $U_{d}$ be the open subscheme of $\bar{U}_{d}$ on which

$$
\text { rk } A+\operatorname{rk} B \geqslant d .
$$

Then $U_{d}$ is smooth over $\operatorname{Spec} \mathbb{Z}[t]$ of relative dimension $d^{2}$.

Proof. We first observe that the fibers are smooth of dimension $d^{2}$ : over points with $t \neq 0$, this is clear, as $U_{d}$ is simply isomorphic to $\mathrm{GL}_{d}$; on the other hand, where $t=0$ Strickland [Str82] shows that $U_{d}$ is reduced of dimension $d^{2}$, and if we fix the ranks of $A$ and $B$ (necessarily adding to $d$ ), we obtain an open subset of $U_{d}$ which is an orbit of the action of $\mathrm{GL}_{d} \times \mathrm{GL}_{d}$, and must therefore be smooth.

Thus, it is enough to show that $U_{d}$ is flat over $\operatorname{Spec} \mathbb{Z}[t]$. For this, we appeal to Lemma 4.3 of [HO08], which asserts that it is enough to check that for any base change of $U_{d}$ to Spec $R$ with $R$ a discrete valuation ring, no component of the base change is supported in the special fiber. This then amounts to the assertion that if we are given a discrete valuation ring $R$, and an element $x$ of $R$, that the scheme of pairs of $d \times d$ matrices $A, B$ over $R$ with $A B=B A=x I_{d}$ and with $\operatorname{rk} A+\operatorname{rk} B \geqslant d$ does not have components supported over the closed point of $R$. But if we are given such $A, B$ over the residue field $k$ of $R$, with $\operatorname{rk} A=d_{1}$ and $\operatorname{rk} B=d_{2}$, there are two cases to consider: if $x$ is a unit, then $A$ and $B$ are invertible, so we may choose any lift of $A$ to $R$, and set $B=x A^{-1}$. On the other hand, if $x$ maps to 0 in $k$, then up to change of basis on both sides, we may assume $A$ is diagonal with the first $d_{1}$ diagonal entries equal to 1 , and the remaining entries 0 , and $B$ is diagonal with the first $d-d_{2}=d_{1}$ entries equal to 0 , and the remaining entries equal to 1 . We may then lift to $R$ simply by replacing the diagonal 0 s with $x$. This shows that every point in the closed fiber is in fact contained in a section, yielding the desired statement.

Finally, we recall the relevant theorem on linked Grassmannians from [Oss06].

Theorem A.8. Suppose that $S$ is integral and Cohen-Macaulay, and $\mathscr{E}_{\bullet}$ is an $s$-linked chain on $S$. Then every component of $\operatorname{LG}\left(r, \mathscr{E}_{\bullet}\right)$ has codimension ( $n-$ $1) r(d-r)$ inside $G\left(r, \mathscr{E}_{1}\right) \times_{S} \cdots \times_{S} G\left(r, \mathscr{E}_{n}\right)$, and if $s$ is nonzero, then $\mathrm{LG}\left(r, \mathscr{E}_{\bullet}\right)$ is irreducible.

We are now ready to prove our main theorem. 
Proof of Theorem A.3. Let $T$ be the product of $n-1$ copies of $U_{d}$ over $\operatorname{Spec} \mathbb{Z}[t]$, and let $S_{0}^{\text {univ }}$ be the open subscheme of $T$ on which $\operatorname{ker} A_{i+1} \cap \operatorname{im} A_{i}=(0)$ and $\operatorname{ker} B_{i} \cap \operatorname{im} B_{i+1}=(0)$ for $i=1, \ldots, n-2$. Then we have an $s$-linked chain $\mathscr{E}_{\bullet}$ univ on $S_{0}^{\text {univ }}$ (with $s=t$ ) by taking $n$ copies of the trivial bundle, and using the $A_{i}$ and $B_{i}$ to define our maps. Let $S^{\text {univ }}$ be obtained from $S_{0}^{\text {univ }}$ as in Proposition A.6. We claim that it is enough to prove the theorem for the corresponding linked determinantal locus on $S^{\text {univ }}$. Indeed, given any $S$ and $\mathscr{E}_{\bullet}$, the theorem is local on $S$, so we may assume that the $\mathscr{E}_{i}$ are trivialized, and our $s$-linked chain and subbundles $\mathscr{F}_{1}$ and $\mathscr{F}_{n}$ then induce a morphism to $S^{\text {univ }}$ under which they are obtained as the pullbacks of $\mathscr{E}_{\bullet}$ univ and the universal subbundles. Moreover, under this morphism we have that the linked determinantal locus on $S$ is the preimage of the linked determinantal locus on $S^{\text {univ }}$. Now, by Proposition A.7 we have that $S_{0}^{\text {univ }}$ and hence $S^{\text {univ }}$ is smooth over Spec $\mathbb{Z}[t]$, and hence regular, and it then follows by Theorem 7.1 of [Hoc75] that if every component of the linked determinantal locus in $S^{\text {univ }}$ has codimension at most $r(d-r)$, then the same is true in $S$.

But according to Proposition A.6, the linked determinantal locus in $S^{\text {univ }}$ is the image of the linked Grassmannian $\operatorname{LG}\left(r, \mathscr{E}_{\bullet}\right.$ univ $)$ over $S_{0}^{\text {univ }}$. By Theorem A.8, we know that $\operatorname{LG}\left(r, \mathscr{E}_{\bullet}\right.$ univ $)$ is irreducible of codimension $(n-1) r(d-r)$, and it is clear that it maps generically finitely onto its image in $S^{\text {univ }}$, since for $t \neq 0$ the subbundle $\mathscr{F}_{1}$ uniquely determines all the other subbundles. We thus conclude by Proposition 5.6.5 of [GD65] that the image - that is, the linked determinantal locus - has codimension $r(d-r)$, as desired.

\section{REFERENCES}

[AB] Omid Amini and Matthew Baker, Linear series on metrized complexes of algebraic curves, Mathematische Annalen, to appear.

[BN07] Matthew Baker and Serguei Norine, Riemann-Roch and Abel-Jacobi theory on a finite graph, Advances in Mathematics 215 (2007), no. 2, 766-788.

[CDPR12] Filip Cools, Jan Draisma, Sam Payne, and Elina Robeva, A tropical proof of the Brill-Noether theorem, Advances in Mathematics 230 (2012), no. 2, 759-776.

[EH83] David Eisenbud and Joe Harris, Divisors on general curves and cuspidal rational curves, Inventiones Mathematicae 74 (1983), 371-418.

[EH87] The Kodaira dimension of the moduli space of curves of genus $\geqslant 23$, Inventiones Mathematicae 90 (1987), 359-387.

[EM02] Eduardo Esteves and Nivaldo Medeiros, Limit canonical systems on curves with two components, Inventiones Mathematicae 149 (2002), no. 2, 267-338.

[GD65] Alexander Grothendieck and Jean Dieudonné, Éléments de géométrie algébrique: IV. Étude locale des schémas et des morphismes de schémas, seconde partie, Publications mathématiques de l'I.H.É.S., vol. 24, Institut des Hautes Études Scientifiques, 1965.

[GH80] Phillip Griffiths and Joseph Harris, On the variety of special linear systems on a general algebraic curve, Duke Mathematical Journal 47 (1980), 233-272.

[Gie82] D. Gieseker, Stable curves and special divisors: Petri's conjecture, Inventiones Mathematicae 66 (1982), 251-275.

[Goe01] Ulrich Goertz, On the flatness of models of certain Shimura varieties of PEL-type, Mathematische Annalen 321 (2001), 689-727.

$\left[\mathrm{GPHH}^{+}{ }^{12}\right]$ Luis García-Puente, Nickolas Hein, Christopher Hillar, Abraham Martín del Campo, James Ruffo, Frank Sottile, and Zach Teitler, The secant conjecture in the real Schubert calculus, Experimental Mathematics 21 (2012), no. 3, 252-265.

[HM82] Joe Harris and David Mumford, On the Kodaira dimension of the moduli space of curves, Inventiones Mathematicae 67 (1982), 23-88.

[HO08] David Helm and Brian Osserman, Flatness of the linked Grassmannian, Proceedings of the AMS 136 (2008), no. 10, 3383-3390. 
[Hoc75] Melvin Hochster, Big Cohen-Macaulay modules and algebras and embeddability in rings of Witt vectors, Queen's Papers on Pure and Applied Math 42 (1975), 106-195.

[Kle76] Steven L. Kleiman, $r$-special subschemes and an argument of Severi's, Advances in Mathematics 22 (1976), no. 1, 1-31.

[Mai98] Laila Maino, Moduli space of enriched stable curves, PhD thesis, Harvard University, 1998.

[MO] John Murray and Brian Osserman, Linked determinantal loci and limit linear series, preprint.

[Oss06] Brian Osserman, A limit linear series moduli scheme, Annales de l'Institut Fourier 56 (2006), no. 4, 1165-1205.

[Oss13] _ Relative dimension of morphisms and dimension for algebraic stacks, preprint, 2013.

[Oss14a] Dimension counts for limit linear series on curves not of compact type, preprint, 2014.

[Oss14b] Limit linear series and the Amini-Baker construction, in preparation, 2014.

[Oss14c] _ Limit linear series moduli stacks in higher rank, preprint, 2014.

[OT14] Brian Osserman and Montserrat Teixidor i Bigas, Linked alternating forms and linked symplectic Grassmannians, International Mathematics Research Notices 2014 (2014), no. 3, 720-744.

[Str82] Elisabetta Strickland, On the conormal bundle of the determinantal variety, Journal of Algebra 75 (1982), no. 2, 523-537.

[Tei91] Montserrat Teixidor i Bigas, Brill-Noether theory for stable vector bundles, Duke Mathematical Journal 62 (1991), no. 2, 385-400. 